\title{
Title: Phytochromes measure photoperiod in Brachypodium
}

\author{
Authors: \\ Mingjun Gao ${ }^{1}$, Feng Geng ${ }^{1}$, Cornelia Klose ${ }^{2}$, Anne-Marie Staudt ${ }^{2}$, He Huang ${ }^{3}$, Duy Nguyen ${ }^{1}$, \\ Hui Lan ${ }^{1}$, Todd C. Mockler ${ }^{3}$, Dmitri A. Nusinow ${ }^{3}$, Andreas Hiltbrunner ${ }^{2,4}$, Eberhard \\ Schäfer ${ }^{2,5}$, Philip A. Wigge ${ }^{1,6,7}$ and Katja E. Jaeger ${ }^{1,6 *}$
}

\section{Affiliations:}

${ }^{1}$ Sainsbury Laboratory, University of Cambridge, 47 Bateman St., Cambridge CB2 1LR, UK.

${ }^{2}$ Institut für Biologie II, University of Freiburg, Schaenzlestr. 1, D-79104 Freiburg, Germany.

${ }^{3}$ Donald Danforth Plant Science Center, St. Louis, MO 63132, USA

${ }^{4}$ Signalling Research Centres BIOSS and CIBSS, University of Freiburg, Schaenzlestr. 18, 79104 Freiburg, Germany

${ }^{5}$ BIOSS Centre for Biological Signalling Studies, University of Freiburg, Schaenzlestr. 18, 79104 Freiburg, Germany

${ }^{6}$ Leibniz-Institut für Gemüse- und Zierpflanzenbau, Theodor-Echtermeyer-Weg 1, 14979 Großbeeren, Germany

${ }^{7}$ Institute of Biochemistry and Biology, University of Potsdam, 14476 Potsdam, Germany

*Correspondence to: jaeger@igzev.de

\section{Summary}

Daylength is a key seasonal cue for animals and plants. In cereals, photoperiodic responses are a major adaptive trait, and alleles of clock genes such as PHOTOPERIOD DEPENDENT1 (PPD1) and EARLY FLOWERING3 (ELF3) have been selected for in breeding barley and wheat for more northern latitudes (Faure et al., 2012; Turner, Beales, Faure, Dunford, \& Laurie, 2005). How monocot plants sense photoperiod and integrate this information into growth and development is not well understood. We show that in Brachypodium distachyon, phytochrome $\mathrm{C}$ (phyC) acts as a molecular timer, directly communicating information to the circadian clock protein ELF3. In this way, ELF3 levels integrate night length information. ELF3 is a 
central regulator of photoperiodism in Brachypodium, and elf3 mutants display a constitutive long day transcriptome. Conversely, conditions that result in higher levels of ELF3 suppress long day responses. We are able to show that these effects are direct, as ELF3 and phyC occur in a common complex, and they associate with the promoters of a number of conserved regulators of photoperiodism, including PPD1. Consistent with observations in barley, we are able to show that PPD1 overexpression accelerates flowering in SD and is necessary for rapid flowering in response to LD. These findings provide a conceptual framework for understanding observations in the photoperiodic responses of key crops, including wheat, barley and rice.

\section{Introduction}

Flowering is a major developmental transition, and plants have evolved pathways to flower in response to seasonal cues to maximise their reproductive fitness (Song, Shim, Kinmonth-Schultz, \& Imaizumi, 2015). Photoperiod provides a key seasonal cue, and in temperate climates, long photoperiods serve as a signal of spring and summer, accelerating flowering in many plants. In Arabidopsis thaliana, long days result in the stabilization of the floral activator CONSTANS (CO), which activates the expression of the florigen encoding gene FLOWERING LOCUS T (FT) (Hayama et al., 2017). Temperate grasses, such as Brachypodium, barley and wheat also induce flowering through the induction of $F T$-related genes, however there are differences in the signalling pathways activating $F T$ expression.

The major regulator of natural variation in photoperiod responsiveness in barley is the transcriptional regulator PHOTOPERIOD DEPENDENT1 (Hv-Ppd1), first identified as dominant allele that accelerates flowering under short day conditions, making plants photoperiod insensitive (Turner et al., 2005). Analyses of ppd1 alleles indicate that promoter insertions and deletions have played a major role modulating $P P D 1$ expression, revealing a 95 bp region within the promoter that is conserved between wheat, barley and Brachypodium (Seki et al., 2013; Wilhelm, Turner, \& Laurie, 2009). It has been hypothesized that a photoperiod dependent repressor may 
bind this 95 bp region short days to inhibit flowering. $P p d-H 1$ also influences leaf size, a trait which is under photoperiod control, consistent with $P p d-H 1$ being a key output of the photoperiod pathway in grasses (Digel et al., 2016). The evening complex (EC), an integral component of the circadian clock, is also a key regulator of photoperiodism in grasses. The early maturity 8 (eam8) allele in barley confers early flowering in SD, and encodes the barley ortholog of EARLY FLOWERING3 (ELF3) (Faure et al., 2012), and in wheat, Earliness Per Se (eps) also confers early flowering and is likely caused by mutation in an ELF3 related gene (Alvarez, Tranquilli, Lewis, Kippes, \& Dubcovsky, 2016). Similarly, eam10 encodes HvLUX, and is necessary for correctly responding to photoperiod (Campoli et al., 2013), while PHYTOCLOCK (LUX) alleles also confer early flowering in wheat (Mizuno et al., 2016).

Unlike in Arabidopsis, where phytochromes repress flowering, PhyC is an essential inducer of flowering in Brachypodium (Woods, Ream, Minevich, Hobert, \& Amasino, 2014), and interfering with phy $C$ in barley and wheat also greatly delays flowering, indicating that phyC is an essential input for photoperiodism (Chen et al., 2014; Nishida et al., 2013). In barley, the allele eam5, which shows constitutively early flowering, is associated with a mutation in the GAF domain of phyC. Since the GAF domains of phytochromes influence their behaviour, this suggests that the interconversion of phyC between the Pfr and Pr states may be important in floral regulation (Pankin et al., 2014). Finally, phyC-1 in Brachypodium also shows additional photoperiod phenotypes such as leaf morphology differences as well as flowering time (Woods et al., 2014) indicating a major role for phytochrome signalling, not just in flowering but photoperiod responses in general. How the EC and PPD1 influence flowering, and how phyC conveys photoperiod information to these regulators is however not well understood.

\section{Results}

To determine if the role of ELF3 in flowering is conserved in Brachypodium, we created loss of function alleles in ELF3. elf3-1 plants show constitutive early flowering, largely independent of photoperiod, indicating that ELF3 is necessary for 
responding to photoperiod (Fig. 1A-C). We find that ELF3 overexpressing plants show delayed flowering in long days, suggesting that ELF3 is necessary and sufficient to transmit photoperiodic signals in Brachypodium (Fig. 1D and E). To understand how ELF3 may be controlling photoperiodic responses in Brachypodium we performed affinity purification coupled with mass spectrometry to identify the ELF3 protein interactome. Consistent with the evening complex being conserved between Arabidopsis and monocots (Huang et al., 2017), ELF4 and LUX are detected as ELF3 interactors (Fig. 1F; Supplementary Dataset S1). The identification of two TOPLESS (TPL)-related proteins suggests a mechanism by which the evening complex represses gene expression. Photoperiodism in Arabidopsis is also mediated by the repression of $F T$ and $C O$ by a TPL containing transcriptional complex, indicating this may be a common mechanism to achieve photoperiodic gene expression (Goralogia et al., 2017). Photoperiodism requires light perception, and we identified the light sensing phytochromes phyB and phy $\mathrm{C}$ as ELF3 interactors. Brachypodium contains three phytochromes, and we therefore investigated the extent to which phytochromes are necessary for photoperiodism. phy C-4 does not flower under LD, consistent with previous reports (Fig. S1A and B) (Woods et al., 2014), while phyA-1 and phyB-1 both show delayed responses to LD (Fig. S1C-F). These results suggest that phytochromes act in the same pathway as ELF3.

Flowering is a complex trait, and it is possible that the perturbation of the floral transition we observe in phyC-4 and elf3-1 is indirect. We therefore compared the transcriptomes of elf3-1 and phyC-4 with that of wild-type plants in response to LD (Fig. 2, Fig. S2, Fig. S3 and Supplementary dataset S2). A large number of genes are induced in the afternoon and evening by LD in two-week old seedlings, and multiple clusters show enhanced expression in response to photoperiod (particularly $8,13,19,22$ and 26). These LD expressed clusters are also induced in two-week old elf3-1 seedlings grown in SD, indicating that ELF3 is necessary for photoperiod dependent expression of the transcriptome. This is also apparent when comparing the elf3-1 transcriptome under LD with elf3-1 under SD, here there is very little 
difference in expression, indicating that elf3-1 has a photoperiod insensitive transcriptome. The constitutive early flowering behaviour of elf3-1 plants is therefore reflected in these plants having a constitutive LD transcriptome even when grown under SD. (Fig. S3). Two clusters (4 and 34) appear to reflect mainly developmental age, and they are primarily differentially expressed in elf3-1 at week 3 compared to week 2. Cluster 34 contains FT1, FT2, BdAP1 and BdMAD5, all indicators of flowering, consistent with these clusters containing downstream targets (Supplementary dataset S3). In contrast to elf3-1, phyC-4 plants show the opposite phenotype, with large scale repression of those genes that are induced by LD in wildtype (Fig. 2 and Fig. S3). Many of the photoperiod response clusters, which are differentially affected by elf3-1 and phyC-4 contain classical photoperiod and circadian genes. Cluster 8 displays a characteristic evening complex target gene behaviour, being more expressed in elf3-1 at the end of the day. This cluster contains classical clock genes such as GI, PPD1 and BdLNK1, and is enriched for genes involved in photosynthesis, as has been observed for EC targets in Arabidopsis (Fig. S4) (Ezer et al., 2017). Cluster 22 shows a strong photoperiod response, and elf3-1 mimics this pattern of expression with repression in the morning, while phyC-4 shows the opposite behaviour. This cluster has photoperiod and clock-related genes including PIF3, CCA1, RVE6 and RVE7. These results indicate that ELF3 is necessary for repressing the LD transcriptome while $P H Y C$ is required for activating gene expression in response to inductive photoperiods.

To understand how ELF3 influences photoperiodic gene expression we identified ELF3 target genes by ChIP-seq. Consistent with what has been seen in Arabidopsis, we observe binding of ELF3 at the promoters of clock genes such as GIGANTEA (GI) (Fig. 3A). We observe that the EC appears to be photoperiod responsive since ELF3 occupancy on promoters is strongly affected by the time of day and the light regime. Binding is maximal in short days during and at the end of the night, but rapidly declines in response to light. Continuous light or LD greatly reduce ELF3 occupancy (Fig. 3B). We used this dynamic change in ELF3 occupancy as a signature to identify putative biologically relevant ELF3 ChIP-seq peaks. In this way, 
161 we identified 94 genes associated with time of day responsive ELF3 ChIP-seq peaks (Supplementary Dataset S4). About 1/3 of these genes associated with ELF3 ChIPseq peaks show an ELF3 dependent expression pattern (Fig. 3C). In Arabidopsis ELF3 functions as a transcriptonal repressor (Ezer et al., 2017), and we observe the same behaviour in Brachypodium, as ELF3 bound genes are strongly up-regulated at the end of the day in elf3-1 (Fig. S5). Many genes bound by ELF3 are induced by LD as well as elf3-1, while they are repressed in phyC-4 (Fig. 3D and Fig. S6), consistent with ELF3 being a central regulator of photoperiod controlled gene expression.

Flowering is controlled by $F T$ (florigen) genes (Wigge, 2011), and we find that $F T 1$ shows the strongest response and absolute expression level in response to inductive photoperiods. Ectopic overexpression of FT1 is sufficient to restore flowering in SD (Fig. S7) suggesting the induction of this gene in response to long photoperiod is important in the activation of flowering. Although we see some evidence for binding of ELF3 at the promoter of FT2 (Fig. S8), no binding at FT1 is observed, suggesting that ELF3 transmits photoperiodism signals to FT1 indirectly.

To identify potential ELF3 bound genes that may act as activators of $F T 1$, we searched for transcripts encoding transcriptonal regulators induced by LD, age and elf3-1 and repressed in phyC-4. PPD1/PRR37 shows a particularly strong response, and we observe a correlation of FT1 and PPD1 expression with age (Fig. S6 and S9). The binding of ELF3 to the PPD1 promoter is also responsive to photoperiod (Fig. S10 and 11). Consistent with this apparent role for ELF3 in controlling PPD1/PRR37 expression, ELF3-OX plants show a strong down-regulation of PPD1/PRR37 transcript levels, which is accompanied by a loss of FT1 expression (Fig. S12).

By genome-editing we created a loss of function allele, ppd1-1, which we confirmed as being late flowering in LD, while expression of PPD1 under the UBIQUITIN promoter (PPD1-OX) is sufficient to activate flowering in SD (Fig. 3E-G). Consistent 
with this phenotype, we observe a strong up-regulation of $F T 1$ in PPD1-OX, as well as that of FT2 and AP1 (Fig. S13). Altering PPD1 activity also affects a number of photoperiod responsive transcripts, but not as many as seen for elf3-1 and phyC-4, consistent with PPD1/PRR37 being downstream of these genes in the photoperiod pathway (Fig. S6). Since ppd1-1 does not have as strong a flowering phenotype as phyC-4 this indicates that other genes contribute to the activation of FT1 in parallel to PPD1. Possible candidates include four genes encoding B-box Proteins (BBX), including the photoperiod regulator CONSTANS (CO) as well as the Brachypodium gene related to GHD7 that controls flowering in rice. Additionally, 4 Cycling DOF (CDF) encoding genes are also directly bound by ELF3 (Supplementary Table S4).

Taken together, these results suggest a model whereby ELF3 represses the expression of PPD1 and other flowering regulators, and under inductive photoperiods ELF3 levels decline, enabling the up-regulation of these transcriptional activators and the activation of FT genes and flowering. The expression of ELF3 however is largely constant and does not show significant circadian variation, and it is expressed in both SD and LD and is unchanged in phyC-4 compared to wild-type (Fig. S14). This suggests that the regulation of ELF3 may be post-translational. Consistent with this hypothesis, the late flowering phenotype of ELF3-OX is sensitive to light exposure (Fig. 4A and B). While ELF3-OX plants are very late flowering in LD, continuous light (LL) is able to accelerate flowering, consistent with our observations for the degree of binding of ELF3 to target promoters (Fig. 3B; Fig. S10 and S11). We therefore hypothesized that ELF3 protein levels are light sensitive. Indeed, ELF3 protein accumulates at the end of the night to high levels under SD, and is rapidly degraded upon exposure to light. A similar pattern is seen under LD, but the levels of ELF3 are lower (Fig. 4C and D, Fig. S15).

Since phyC-4 transcriptionally resembles a plant with elevated ELF3 signaling, this suggests that phyC may be the major light receptor controlling ELF3 activity. To determine if this occurs via a direct mechanism, we performed ChIP-seq of phyC. In Arabidopsis, phyB binds to target genes to modulate their expression (Jung et al., 
2016), and we investigated if this might be true for phyC in Brachypodium. We observe coincidence between ELF3 and phyC ChIP-seq peaks for many key genes such as LUX (Fig. 4E and Fig. S16; Supplementary Dataset S5). Phytochromes have been observed to interact with ELF3 in other systems, so this finding is consistent with these observations and suggests phyC may counteract the ability of ELF3 to repress its targets.

In Arabidopsis, photoperiod is measured through the interaction of phototransduction pathways with the circadian rhythm, and flowering is activated when light corresponds with a sensitive phase of the circadian cycle (Song et al., 2015). This has been demonstrated using non-24 h light-dark cycles (T-cycles), an approach first described by Nanda and Hamner (Nanda \& Hamner, 1958). Flowering does not correlate with the length of the light or dark for non-24 h T-cycles, indicating that Arabidopsis does not measure night or day length alone to determine photoperiodism (Roden, Song, Jackson, Morris, \& Carre, 2002). By contrast, other plants such as Xanthium measure night length to determine photoperiodism (Hamner, 1940;

\section{Thomas \& Vince-Prue, 1997). Our results so far indicate that night-length} measurement via the integration of Pfr levels is an important mechanism in Brachypodium. This predicts that, unlike for Arabidopsis, modulating night-length alone will have the largest effect on flowering in Brachypodium. To test this, we grew plants under a range of T-cycles. As expected SD control plants under 12L:12D do not flower, while LD (20L:4D) plants do (Fig. 4F and G and Fig. S17). Combining a long day with a long night (20L:12D) also prevents flowering, consistent with nightlength being the main determinant of the photoperiodic response in Brachypodium. $A$ long day is not required for flowering, since plants grown under 12L:4D cycles are early flowering. In Arabidopsis, growing plants under different T-cycles alters the phase of the circadian rhythm with the light-dark cycle. For example plants grown in 10L:20D actually flower earlier than plants grown under 10L:14D, although they both experience the same duration of day length. To investigate whether some of the effects we observe might be due to alterations in the phasing of the external daynight cycle with endogenous rhythms, we grew plants under 12L:20D, extending the 
usual night by $8 \mathrm{~h}$ and thereby altering the phasing of the circadian clock compared to $12 \mathrm{~L}: 12 \mathrm{D}$ grown plants. These plants also display a SD phenotype and do not flower, suggesting that absolute night-length plays a major role in determining photoperiodic responses in Brachypodium (Fig. S17).

Since phytochromes respond to light rapidly, we predicted that brief exposure to light during the dark period ("night-break") would be sufficient to overcome the repressive effects of long nights on flowering. In the case of rice, the introduction of a nightbreak prevents flowering (Ishikawa, Shinomura, Takano, \& Shimamoto, 2009), while in wheat flowering can be activated by night-break (Pearce et al., 2017). Consistent with a role for phytochromes in mediating a night-length signal, night-breaks are sufficient to restore flowering in SD grown plants (Fig. S18).

\section{The results so far are consistent with phytochromes playing a role in perceiving night} length and coordinating the photoperiod transcriptome via ELF3. Differential protein stability of phyC or PPD1/PRR37 would represent a potential mechanism to achieve photoperiodism. However we observe constantly levels of these proteins when they are overexpressed regardless of ZT time, suggesting modulating phyC protein levels is not key (Fig. S19). Since phytochromes in the active, Pfr, state slowly revert to the inactive Pr state in the dark (thermal or dark reversion), we hypothesized that this presents a mechanism for measuring the length of the night. Under long photoperiods the dark period may be insufficient for phyC Pfr to be depleted, with the result that ELF3 cannot accumulate to a high level. Extending the night period in short days however may enable phyC Pfr to become depleted, allowing the accumulation of repressive ELF3. To test this, we measured the dark reversion dynamics of Brachypodium phyC by overexpressing the gene in Brachypodium and Arabidopsis seedlings. In both cases, we observe similar reversion rates, and the dark reversion of phyC Pfr has a half-life of $8.3 \mathrm{~h}$ in Brachypodium (Fig. $\mathbf{4 H}$; Supplementary Dataset S6). Under our long day conditions therefore, 72 \% of Pfr remains at the end of the night, while in short day conditions only $37 \%$ of $\mathrm{Pfr}$ is present at ZTO. This is consistent with the differences we observe in the binding of 
ELF3 to promoters in response to photoperiod, and suggests a model whereby the levels of Pfr provide a readout of night-length and communicate this to a central regulator of photoperiodism, ELF3 (Fig. S20).

\section{Discussion}

Photoperiodism provides plants with important seasonal information to control their behaviour. As well as fundamental differences, such as long and short day plants, there is also considerable variation in the relative contribution of different factors to photoperiod sensitivity. Arabidopsis measures day-length to activate flowering (Hayama et al., 2017), and the phasing of the circadian rhythm with the external lightdark cycle is particularly important (Roden et al., 2002). In Brachypodium, wheat, rice and poplar, night-length is a critical determinant of photoperiodism (Ishikawa et al., 2005; Jos Ramos-Sá nchez et al., 2019; Pearce et al., 2017). We are able to show that phyC plays a central role in this process, and the dark reversion rate of $\mathrm{Pfr}$ is consistent with a role for phyC as a "molecular hourglass" (Borthwick \& Hendricks, 1960). While proposed about 60 years ago, this model was discounted on discovering a circadian variation in sensitivity to far red-light pulses during extended darkness (Cumming, Hendricks, \& Borthwick, 1965). Our finding that phytochromes directly modulate the activity of the circadian component ELF3 suggests a mechanism to reconcile these observations. It will be interesting to see if the phyCELF3 signalling module is widely conserved to control photoperiodism, since this appears to be the case in rice (Itoh, Tanaka, \& Izawa, 2018), and an ELF3 ortholog also controls photoperiodism in Pea (Rubenach et al., 2017).

Light quality at dusk varies seasonally (Hughes, Morgan, Lambton, Black, \& Smith, 1984; Linkosalo \& Lechowicz, 2006), and in Aspen phytochrome signaling controls growth cessation and budset during autumn (Olsen et al., 1997). The ability of phytochromes to integrate changes in both spectral quality and photoperiod as well as temperature (Jung et al., 2016; Legris et al., 2016) may represent a robust 


\section{Acknowledgements}

We thank Richard Amasino and Daniel Woods for sharing Brachypodium mutant lines. The work in KEJ's laboratory is supported by the Leibniz Association. KEJ was supported by a fellowship from the Gatsby Foundation. PAW's laboratory is supported by the Leibniz Association and the Leibniz IGZ.

\section{References}

Alvarez, M. A., Tranquilli, G., Lewis, S., Kippes, N., \& Dubcovsky, J. (2016). Genetic and physical mapping of the earliness per se locus Eps-A m 1 in Triticum monococcum identifies EARLY FLOWERING 3 (ELF3) as a candidate gene. Functional \& Integrative Genomics, 16(4), 365-382. https://doi.org/10.1007/s10142-016-0490-3

Borthwick, H. A., \& Hendricks, S. B. (1960). Photoperiodism in Plants. Science (New York, N.Y.), 132(3435), 1223-1228.

Campoli, C., Pankin, A., Drosse, B., Casao, C. M., Davis, S. J., \& von Korff, M. (2013). HVLUX1 is a candidate gene underlying the early maturity 10 locus in barley: phylogeny, diversity, and interactions with the circadian clock and photoperiodic pathways. New Phytologist, 199(4), 1045-1059. https://doi.org/10.1111/nph.12346

Chen, A., Li, C., Hu, W., Lau, M. Y., Lin, H., Rockwell, N. C., ... Dubcovsky, J. (2014). PHYTOCHROME C plays a major role in the acceleration of wheat flowering under long-day photoperiod. Proceedings of the National Academy of Sciences, 111(28), 10037-10044. https://doi.org/10.1073/pnas.1409795111

Cumming, B. G., Hendricks, S. B., \& Borthwick, H. A. (1965). RHYTHMIC FLOWERING RESPONSES AND PHYTOCHROME CHANGES IN A SELECTION OF CHENOPODIUM RUBRUM. Canadian Journal of Botany, 43(7), 825-853. https://doi.org/10.1139/b65-092

Digel, B., Tavakol, E., Verderio, G., Tondelli, A., Xu, X., Cattivelli, L., ... von Korff, M. (2016). Photoperiod-H1 (Ppd-H1) Controls Leaf Size. Plant Physiology, 172(1), 
405-415. https://doi.org/10.1104/pp.16.00977

Ezer, D., Jung, J.-H., Lan, H., Biswas, S., Gregoire, L., Box, M. S., .. Wigge, P. A. (2017). The evening complex coordinates environmental and endogenous signals in Arabidopsis. Nature Plants, 3(7), 17087. https://doi.org/10.1038/nplants.2017.87

Faure, S., Turner, A. S., Gruszka, D., Christodoulou, V., Davis, S. J., von Korff, M., \& Laurie, D. A. (2012). Mutation at the circadian clock gene EARLY MATURITY 8 adapts domesticated barley (Hordeum vulgare) to short growing seasons. Proceedings of the National Academy of Sciences. https://doi.org/10.1073/pnas.1120496109 Goralogia, G. S., Liu, T.-K., Zhao, L., Panipinto, P. M., Groover, E. D., Bains, Y. S., \& Imaizumi, T. (2017). CYCLING DOF FACTOR 1 represses transcription through the TOPLESS co-repressor to control photoperiodic flowering in Arabidopsis. The Plant Journal, 92(2), 244-262. https://doi.org/10.1111/tpj.13649 Hamner, K. C. (1940). Interrelation of Light and Darkness in Photoperiodic Induction. Botanical Gazette, 101(3), 658-687. https://doi.org/10.1086/334903 Hayama, R., Sarid-Krebs, L., Richter, R., Fernández, V., Jang, S., \& Coupland, G. (2017). PSEUDO RESPONSE REGULATORs stabilize CONSTANS protein to promote flowering in response to day length. The EMBO Journal. https://doi.org/10.15252/embj.201693907

Huang, H., Gehan, M. A., Huss, S. E., Alvarez, S., Lizarraga, C., Gruebbling, E. L., ... Nusinow, D. A. (2017). Cross-species complementation reveals conserved functions for EARLY FLOWERING 3 between monocots and dicots. Plant Direct, 1(4), e00018. https://doi.org/10.1002/pld3.18

Hughes, J. E., Morgan, D. C., Lambton, P. A., Black, C. R., \& Smith, H. (1984). Photoperiodic time signals during twilight. Plant, Cell and Environment, 7(4), 269-277. https://doi.org/10.1111/1365-3040.ep11589464

Ishikawa, R., Shinomura, T., Takano, M., \& Shimamoto, K. (2009). Phytochrome dependent quantitative control of $\mathrm{Hd} 3 \mathrm{a}$ transcription is the basis of the night break effect in rice flowering. Genes \& Genetic Systems, 84(2), 179-184. Retrieved from http://www.ncbi.nlm.nih.gov/pubmed/19556711 
Ishikawa, R., Tamaki, S., Yokoi, S., Inagaki, N., Shinomura, T., Takano, M., \& Shimamoto, K. (2005). Suppression of the floral activator Hd3a is the principal cause of the night break effect in rice. Plant Cell, 17(12), 3326-3336. Retrieved from

http://www.ncbi.nlm.nih.gov/entrez/query.fcgi?cmd=Retrieve\&db=PubMed\&dopt $=$ Citation\&list_uids $=16272430$

Itoh, H., Tanaka, Y., \& Izawa, T. (2018). Genetic relationship between phytochromes and OsELF3-1 reveals the mode of regulation for the suppression of phytochrome signaling in rice. Plant and Cell Physiology. https://doi.org/10.1093/pcp/pcy225

Jos Ramos-Sá nchez, A. M., Triozzi, P. M., Alique, D., Wigge, P. A., Allona, I., \& Perales, M. (2019). LHY2 Integrates Night-Length Information to Determine Timing of Poplar Photoperiodic Growth. Current Biology, 29. https://doi.org/10.1016/j.cub.2019.06.003

Jung, J.-H., Domijan, M., Klose, C., Biswas, S., Ezer, D., Gao, M., ... Wigge, P. A. (2016). Phytochromes function as thermosensors in Arabidopsis. Science, 354(6314).

Legris, M., Klose, C., Burgie, E. S., Rojas, C. C. R., Neme, M., Hiltbrunner, A., ... Casal, J. J. (2016). Phytochrome B integrates light and temperature signals in Arabidopsis. Science, 354(6314).

Linkosalo, T., \& Lechowicz, M. J. (2006). Twilight far-red treatment advances leaf bud burst of silver birch (Betula pendula). Tree Physiology, 26(10), 1249-1256.

Retrieved from http://www.ncbi.nlm.nih.gov/pubmed/16815827

Mizuno, N., Kinoshita, M., Kinoshita, S., Nishida, H., Fujita, M., Kato, K., .. Nasuda, S. (2016). Loss-of-Function Mutations in Three Homoeologous PHYTOCLOCK 1 Genes in Common Wheat Are Associated with the Extra-Early Flowering Phenotype. PLOS ONE, 11(10), e0165618.

https://doi.org/10.1371/journal.pone.0165618

Nanda, K. K., \& Hamner, K. C. (1958). Studies on the Nature of the Endogenous Rhythm Affecting Photoperiodic Response of Biloxi Soybean. Botanical Gazette, 120(1), 14-25. https://doi.org/10.1086/335992 
Nishida, H., Ishihara, D., Ishii, M., Kaneko, T., Kawahigashi, H., Akashi, Y., ... Kato, K. (2013). Phytochrome C Is A Key Factor Controlling Long-Day Flowering in Barley. PLANT PHYSIOLOGY, 163(2), 804-814. https://doi.org/10.1104/pp.113.222570

Olsen, J. E., Junttila, O., Nilsen, J., Eriksson, M. E., Martinussen, I., Olsson, O., ... Moritz, T. (1997). Ectopic expression of oat phytochrome A in hybrid aspen changes critical daylength for growth and prevents cold acclimatization. The Plant Journal, 12(6), 1339-1350. https://doi.org/10.1046/j.1365313x.1997.12061339.x

Pankin, A., Campoli, C., Dong, X., Kilian, B., Sharma, R., Himmelbach, A., ... von Korff, M. (2014). Mapping-by-sequencing identifies HvPHYTOCHROME C as a candidate gene for the early maturity 5 locus modulating the circadian clock and photoperiodic flowering in barley. Genetics, 198(1), 383-396.

https://doi.org/10.1534/genetics.114.165613

Pearce, S., Shaw, L. M., Lin, H., Cotter, J. D., Li, C., \& Dubcovsky, J. (2017). NightBreak Experiments Shed Light on the Photoperiod1-Mediated Flowering. Plant Physiology, 174(2), 1139-1150. https://doi.org/10.1104/pp.17.00361

Roden, L. C., Song, H.-R., Jackson, S., Morris, K., \& Carre, I. A. (2002). Floral responses to photoperiod are correlated with the timing of rhythmic expression relative to dawn and dusk in Arabidopsis. Proceedings of the National Academy of Sciences of the United States of America, 99(20), 13313-13318. https://doi.org/10.1073/pnas.192365599

Rubenach, A. J. S., Hecht, V., Vander Schoor, J. K., Liew, L. C., Aubert, G., Burstin, J., \& Weller, J. L. (2017). EARLY FLOWERING3 Redundancy Fine-Tunes Photoperiod Sensitivity. Plant Physiology, 173(4), 2253-2264. https://doi.org/10.1104/pp.16.01738

Seki, M., Chono, M., Nishimura, T., Sato, M., Yoshimura, Y., Matsunaka, H., ... Kato, K. (2013). Distribution of photoperiod-insensitive allele Ppd-A1a and its effect on heading time in Japanese wheat cultivars. Breeding Science, 63(3), 309-316. https://doi.org/10.1270/jsbbs.63.309

Song, Y. H., Shim, J. S., Kinmonth-Schultz, H. A., \& Imaizumi, T. (2015). 
Photoperiodic flowering: time measurement mechanisms in leaves. Annual Review of Plant Biology, 66, 441-464. https://doi.org/10.1146/annurev-arplant043014-115555

Thomas, B., \& Vince-Prue, D. (1997). Photoperiodism in Plants. London: Academic Press.

Turner, A., Beales, J., Faure, S., Dunford, R. P., \& Laurie, D. A. (2005). The pseudoresponse regulator $\mathrm{Ppd}-\mathrm{H} 1$ provides adaptation to photoperiod in barley. Science, 310(5750), 1031-1034. https://doi.org/10.1126/science.1117619 Wigge, P. A. (2011). FT, a mobile developmental signal in plants. Current Biology: CB, 21(9), R374-8. https://doi.org/10.1016/j.cub.2011.03.038 Wilhelm, E. P., Turner, A. S., \& Laurie, D. A. (2009). Photoperiod insensitive PpdA1a mutations in tetraploid wheat (Triticum durum Desf.). Theoretical and Applied Genetics, 118(2), 285-294. https://doi.org/10.1007/s00122-008-0898-9 Woods, D. P., Ream, T. S., Minevich, G., Hobert, O., \& Amasino, R. M. (2014). PHYTOCHROME C Is an Essential Light Receptor for Photoperiodic Flowering in the Temperate Grass, Brachypodium distachyon. Genetics, genetics.114.166785-. https://doi.org/10.1534/genetics.114.166785 
bioRxiv preprint doi: https://doi.org/10.1101/697169; this version posted July 9, 2019. The copyright holder for this preprint (which was not

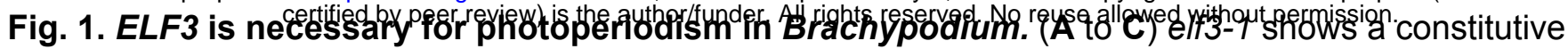
long day flowering phenotype under short day conditions, where wild-type does not flower (NF). (Student's ttest, $\left.{ }^{* *} \mathrm{P}<0.01\right)$. (D to E) Constitutive expression of ELF3 under the UBIQUITIN promoter $\left(U B I_{\text {pro }}\right)$ is sufficient to greatly delay flowering under inductive long day conditions. (Student's t-test, $\left.{ }^{* *} P<0.01\right)$. (F) Curated list of proteins binding ELF3 identified by mass spectrometry. No peptides for these proteins detected in the negative control (YFP-HFC). aAll proteins match 99\% threshold with minimum 2 peptides
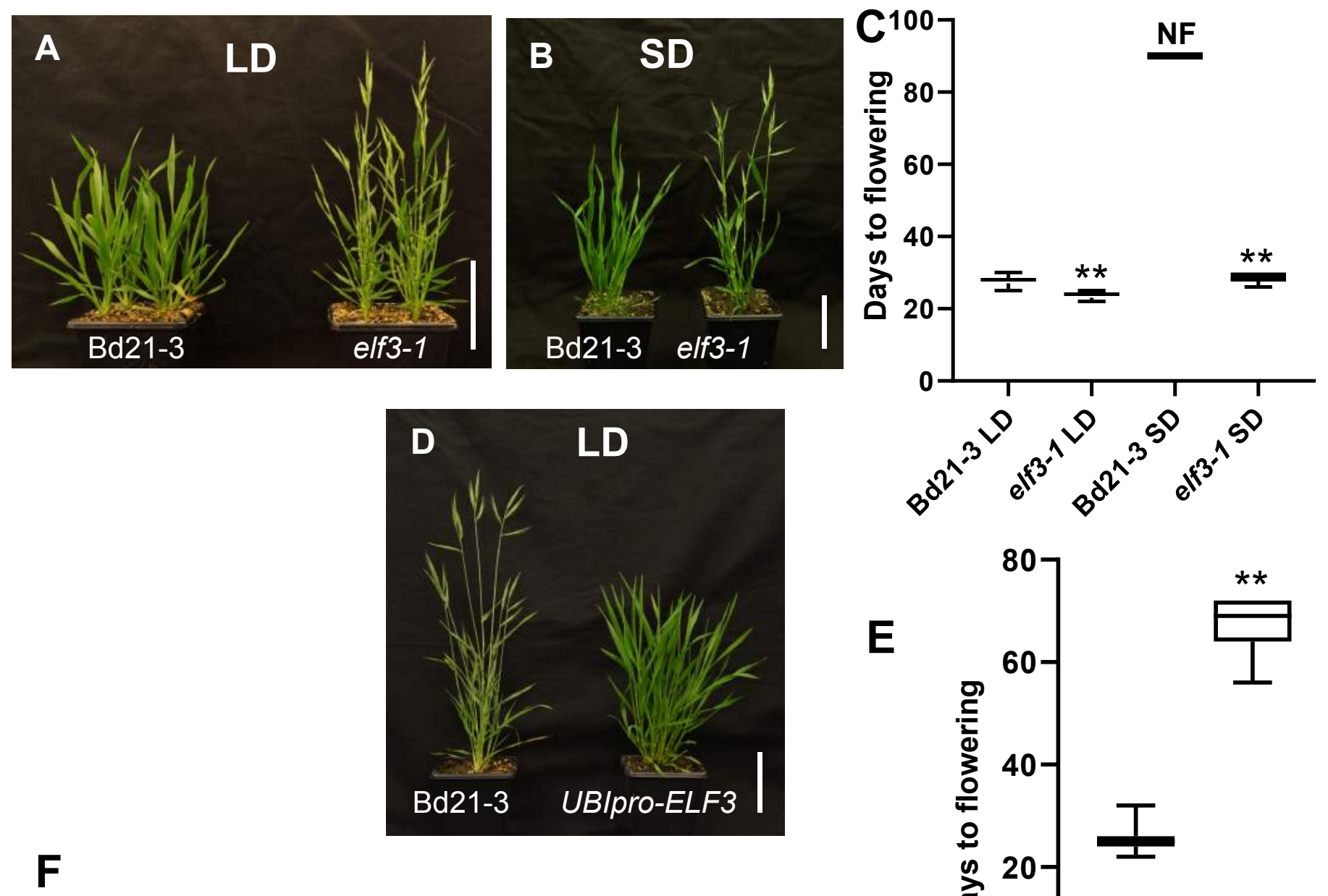

\begin{tabular}{|c|c|c|c|c|}
\hline \multirow[t]{2}{*}{ Gene ID } & \multirow[t]{2}{*}{ Homolog Protein } & \multirow[t]{2}{*}{$\begin{array}{c}\text { Molecular } \\
\text { Weight }\end{array}$} & \multicolumn{2}{|c|}{$\begin{array}{c}\text { Exclusive Unique } \\
\text { Peptide Count/Percen } \\
\text { Coverage }^{\mathrm{a}}\end{array}$} \\
\hline & & & rep1 & rep2 \\
\hline Bradi2g13087 & $\mathrm{ELF}^{\mathrm{b}}$ & $53 \mathrm{kDa}$ & $30 / 58 \%$ & $34 / 60 \%$ \\
\hline Bradi1g64360 & phyB & 129 kDa & $31 / 39 \%$ & $24 / 31 \%$ \\
\hline Bradi1g08400 & phyc & 126 kDa & $2 / 2.8 \%$ & $2 / 2.9 \%$ \\
\hline Bradi3g44580 & LWD1 & $44 \mathrm{kDa}$ & $8 / 40 \%$ & $4 / 20 \%$ \\
\hline Bradi2g09080 & TOPLESS & $125 \mathrm{kDa}$ & $12 / 19 \%$ & $7 / 12 \%$ \\
\hline Bradi3g16250 & TOPLESS-RELATED PROTEIN 2 & $125 \mathrm{kDa}$ & $13 / 22 \%$ & $4 / 7 \%$ \\
\hline Bradi3g57667 & COP1 & $78 \mathrm{kDa}$ & $13 / 26 \%$ & $8 / 18 \%$ \\
\hline Bradi2g46850 & PCH1 & $83 \mathrm{kDa}$ & $10 / 22 \%$ & $5 / 14 \%$ \\
\hline Bradi4g07110 & CDC5, MYB transcription factor & $110 \mathrm{kDa}$ & $15 / 25 \%$ & $3 / 5 \%$ \\
\hline Bradi1g24100 & TIME FOR COFFEE & $171 \mathrm{kDa}$ & $8 / 10 \%$ & $9 / 9.3 \%$ \\
\hline Bradi2g62067 & LUX & $27 \mathrm{kDa}$ & $3 / 31 \%$ & $2 / 20 \%$ \\
\hline Bradi2g48657 & SPA1 & $87 \mathrm{kDa}$ & $11 / 21 \%$ & $7 / 14 \%$ \\
\hline Bradi4g13227 & ELF4 & $13 \mathrm{kDa}$ & $3 / 31 \%$ & $4 / 32 \%$ \\
\hline Bradi1g60090 & ELF4-like & $12 \mathrm{kDa}$ & $2 / 17 \%$ & $3 / 45 \%$ \\
\hline Bradi1g05950 & MLK2/PPK3 & $79 \mathrm{kDa}$ & $8 / 23 \%$ & $3 / 9.6 \%$ \\
\hline Bradi2g51370 & MLKs/PPKs & $78 \mathrm{kDa}$ & $2 / 15 \%$ & $1 / 4.9 \%$ \\
\hline Bradi1g22840 & FRI-like & $67 \mathrm{kDa}$ & $5 / 14 \%$ & $2 / 5.6 \%$ \\
\hline Bradi2g47940 & FVE & $50 \mathrm{kDa}$ & $9 / 41 \%$ & $4 / 16 \%$ \\
\hline
\end{tabular}

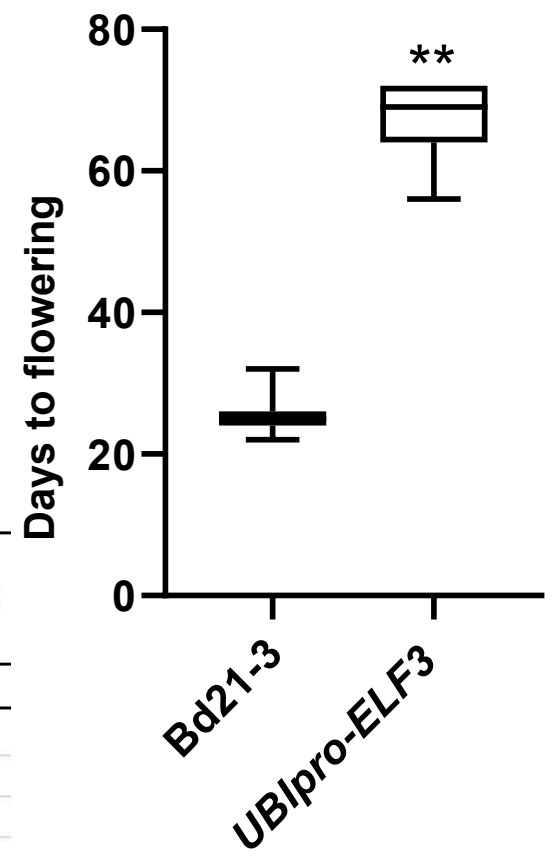


bioRxiv preprint doi: https://doi.org/10.1101/697169; this version posted July 9, 2019. The copyright holder for this preprint (which was not

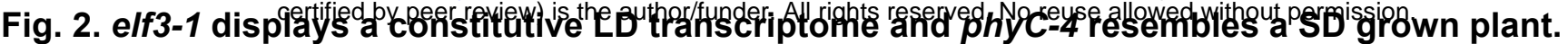

Clustering by gene expression reveals that many of the clusters of genes that respond to LD show a similar behaviour in elf3-1 grown in SD, suggesting that this background has a constitutive LD response. phyC-4 shows the opposite response, resembling a SD plant when grown under LD. Clusters 8, 13, 19, 22 and 26 in particular show up-regulation in response to LD, they are expressed in response to the elf3-1 mutation, and down-regulated in phyC-4. Clusters 4 and 34 appear to reflect developmental changes in response to the floral transition, reflecting the very early flowering phenotype of elf3-1.

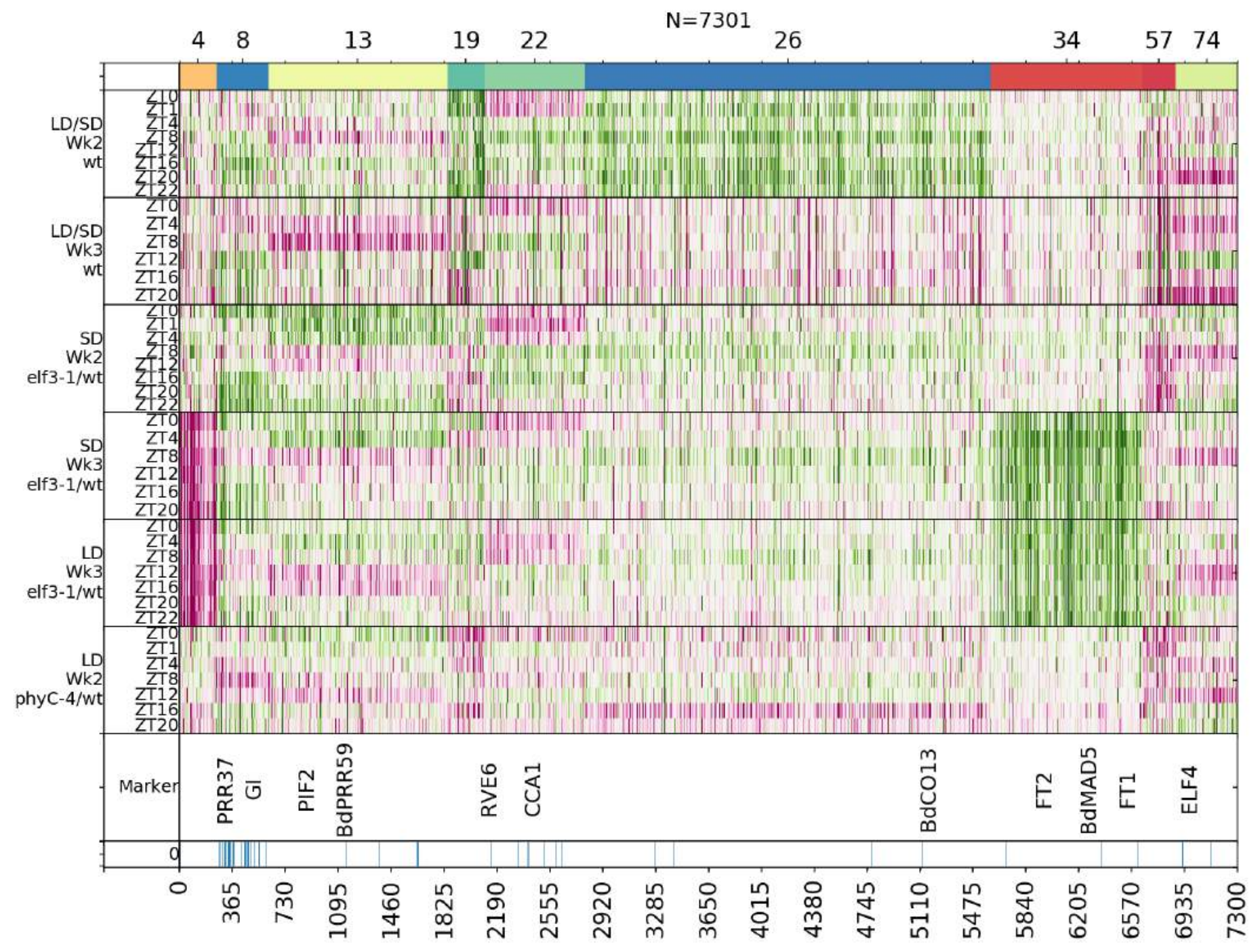


bioRxiv preprint doi: https://doi.org/10.1101/697169; this version posted July 9, 2019. The copyright holder for this preprint (which was not

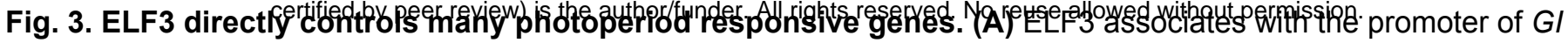
as measured by ChIP-seq. (B) The occupancy of ELF3 on target promoters responds to photoperiod. Binding increases in short days at ZT0 and ZT20, but sharply decreases at ZT4. This is a response to light, since binding at ZT0 and ZT20 is sharply reduced in LD and LL. (C) There are 94 genes differentially bound by ELF3 and about $1 / 3$ of these genes have perturbed expression in elf3-1 (D) These differentially bound direct targets of ELF3 show a high degree of responsiveness to photoperiod. Cluster -1 collects genes without a confident cluster. Genes which are upregulated are shown in green, genes which are downregulated are shown in red and unchanged is depicted in white. (E-G) ppd1-1 is required for photoperiodic acceleration of flowering and UBIpro-PPD1 flower independently of photoperiod

A $\quad 370$
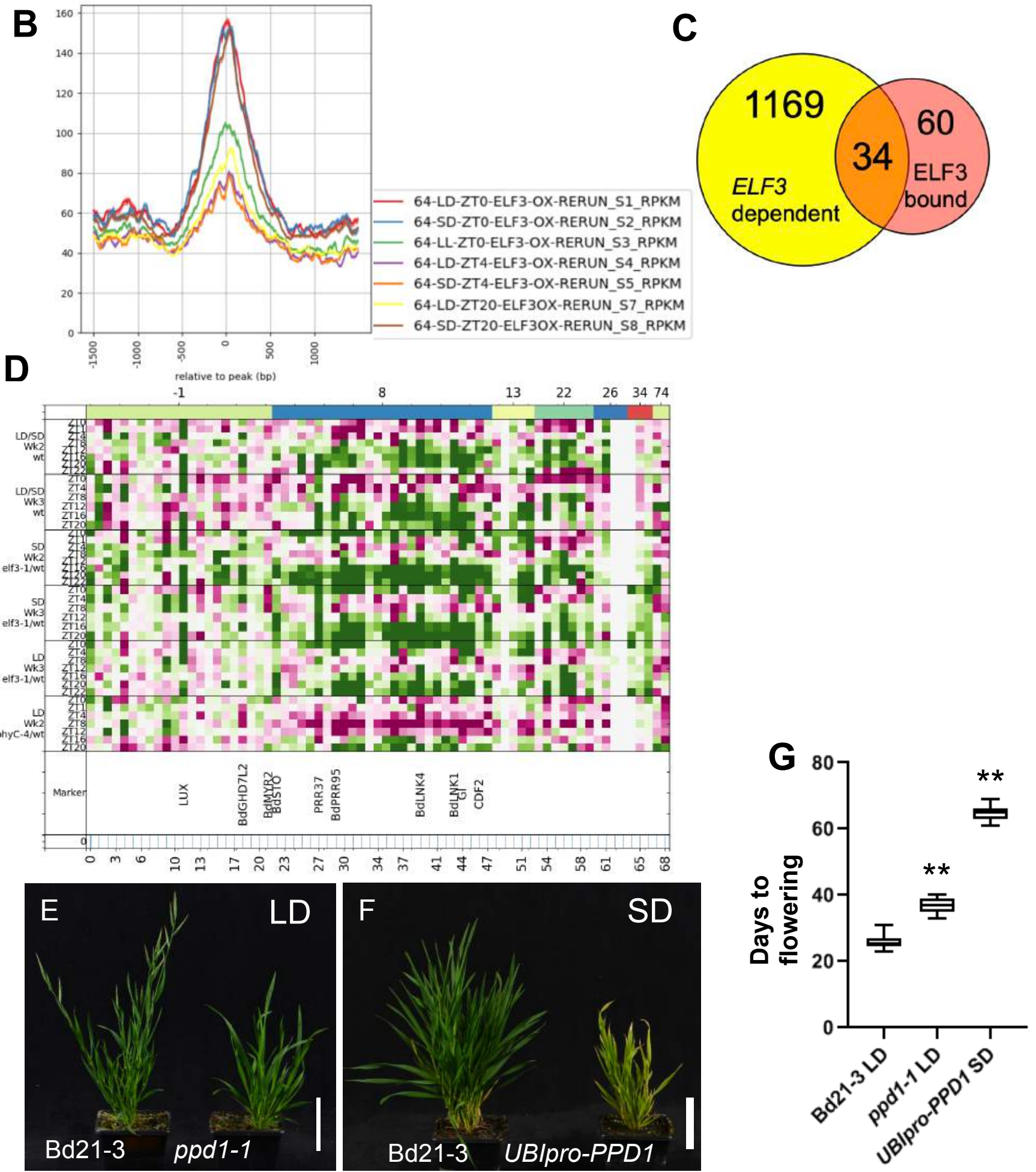
bioRxiv preprint doi: https://doi.org/10.1101/697169; this version posted July 9, 2019. The copyright holder for this preprint (which was not

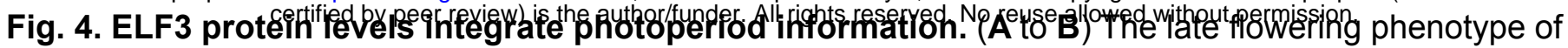
UBIpro-ELF3 in long days is partially suppressed by growth in continuous light. (D and D) ELF3 protein levels accumulate during the night and are rapidly reduced on exposure to light. (E) Overlap between phyC and ELF3 ChIP-seq peaks. (F) phyC dark reversion has a half-life of about $8 \mathrm{~h}$. $\mathbf{G}$ and $\mathbf{H}$ ) Night length but not day length is the key determinant of when plants will flower.
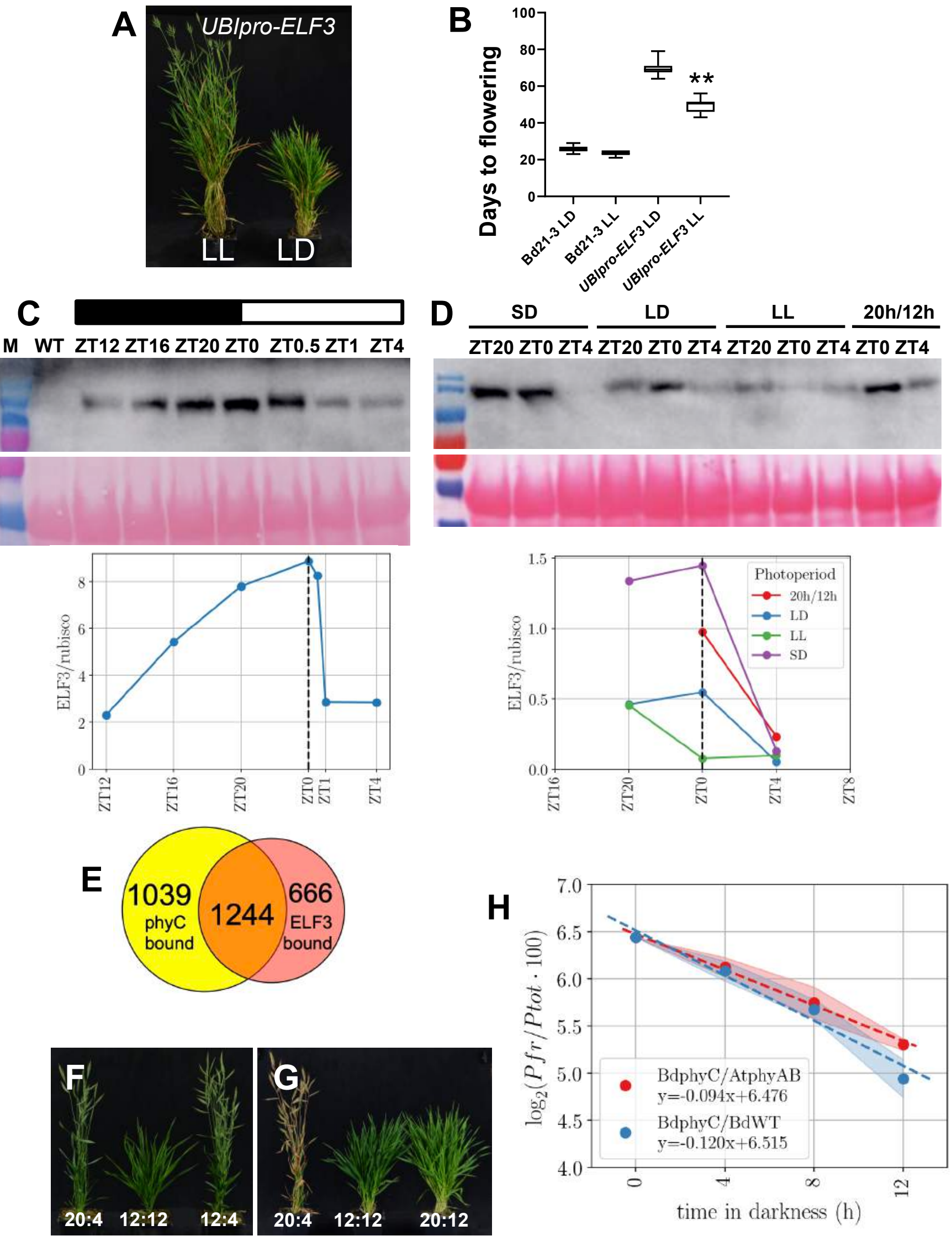
bioRxiv preprint doi: https://doi.org/10.1101/697169; this version posted July 9, 2019. The copyright holder for this preprint (which was not

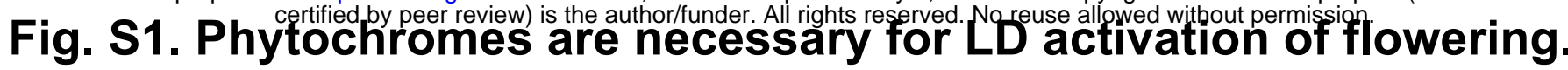
$(\mathbf{A}$ and $\mathbf{B})$ phyC-4 does not flower in inductive conditions. (C and $\mathbf{D}$ ) phy $A-1$ is late flowering in long days. ( $E$ and $\mathbf{F}$ ) phyB-1 is late flowering in long days.
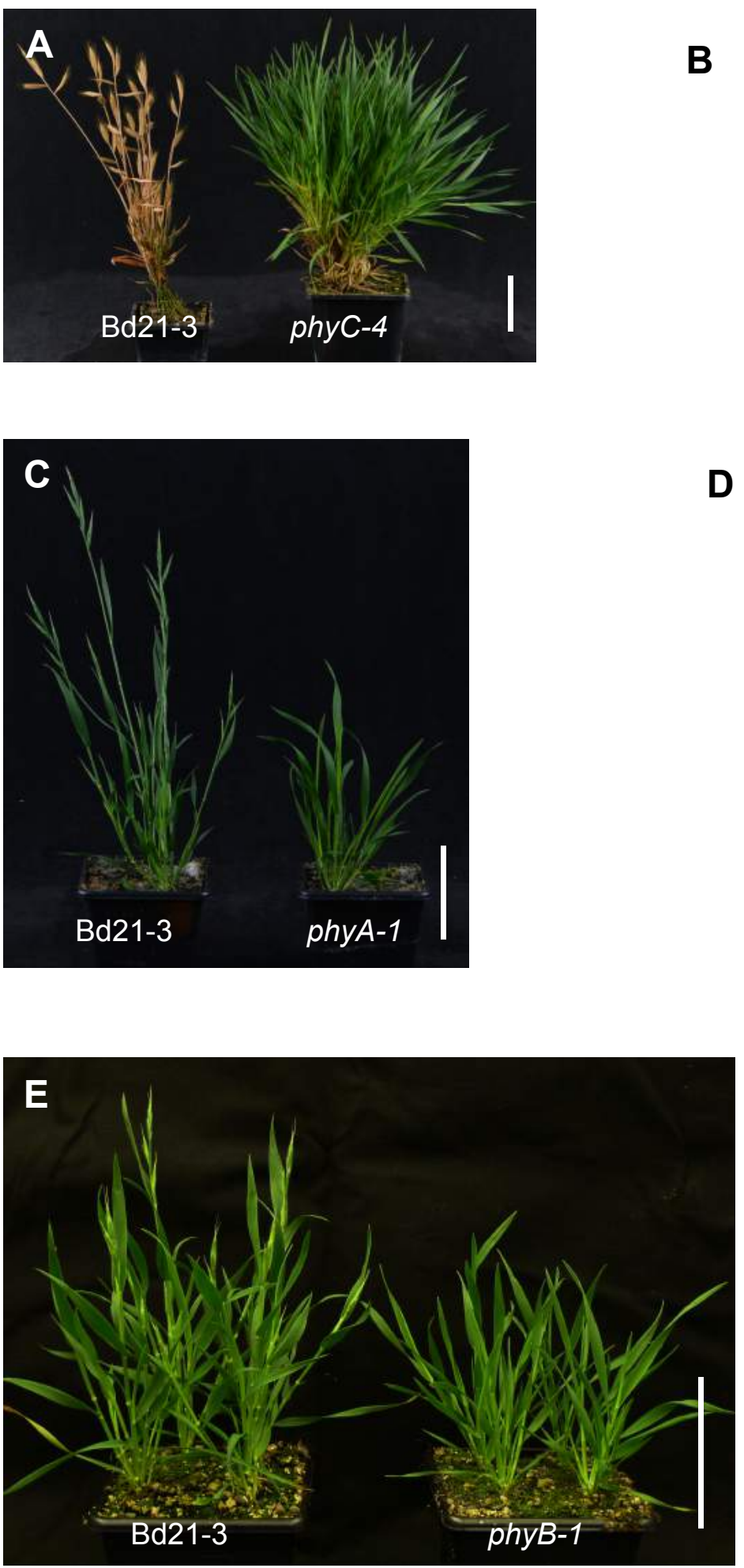

B

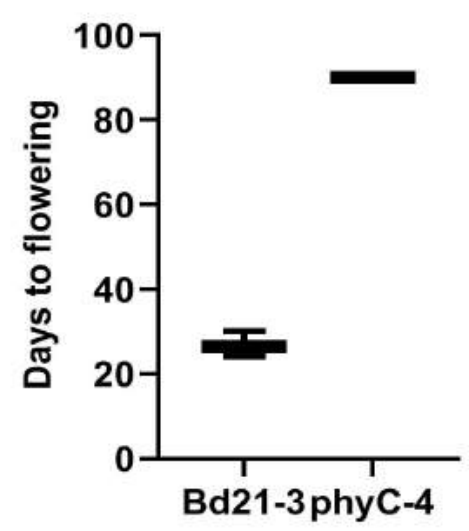

D

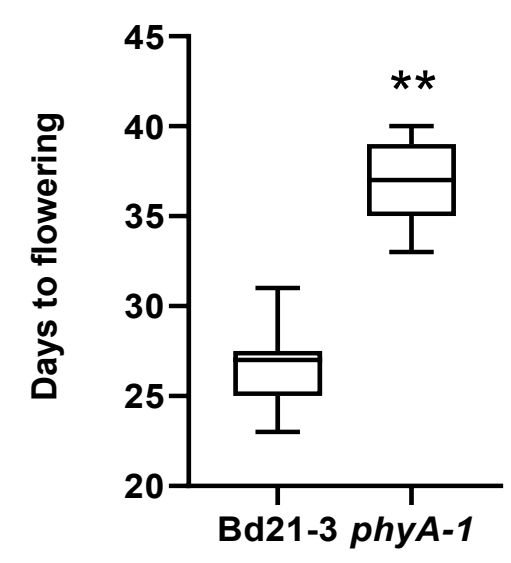

$\mathbf{F}$

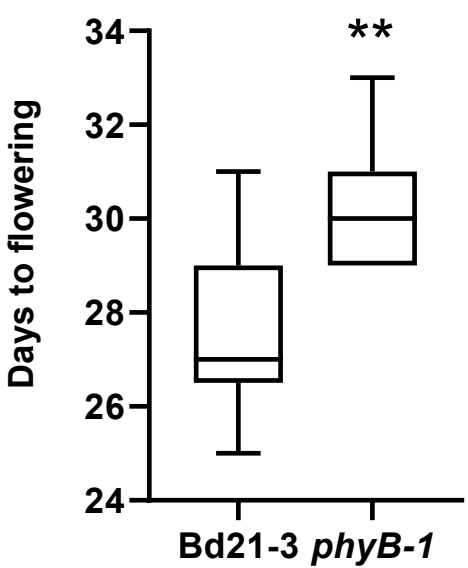


bioRxiv preprint doi: https://doi.org/10.1101/697169; this version posted July 9, 2019. The copyright holder for this preprint (which was not

Fig S2. Sampling scheme for Chiph Schemata for collection points of all RNA-Seq, ChIP-Seq and Western blot series conducted in this study
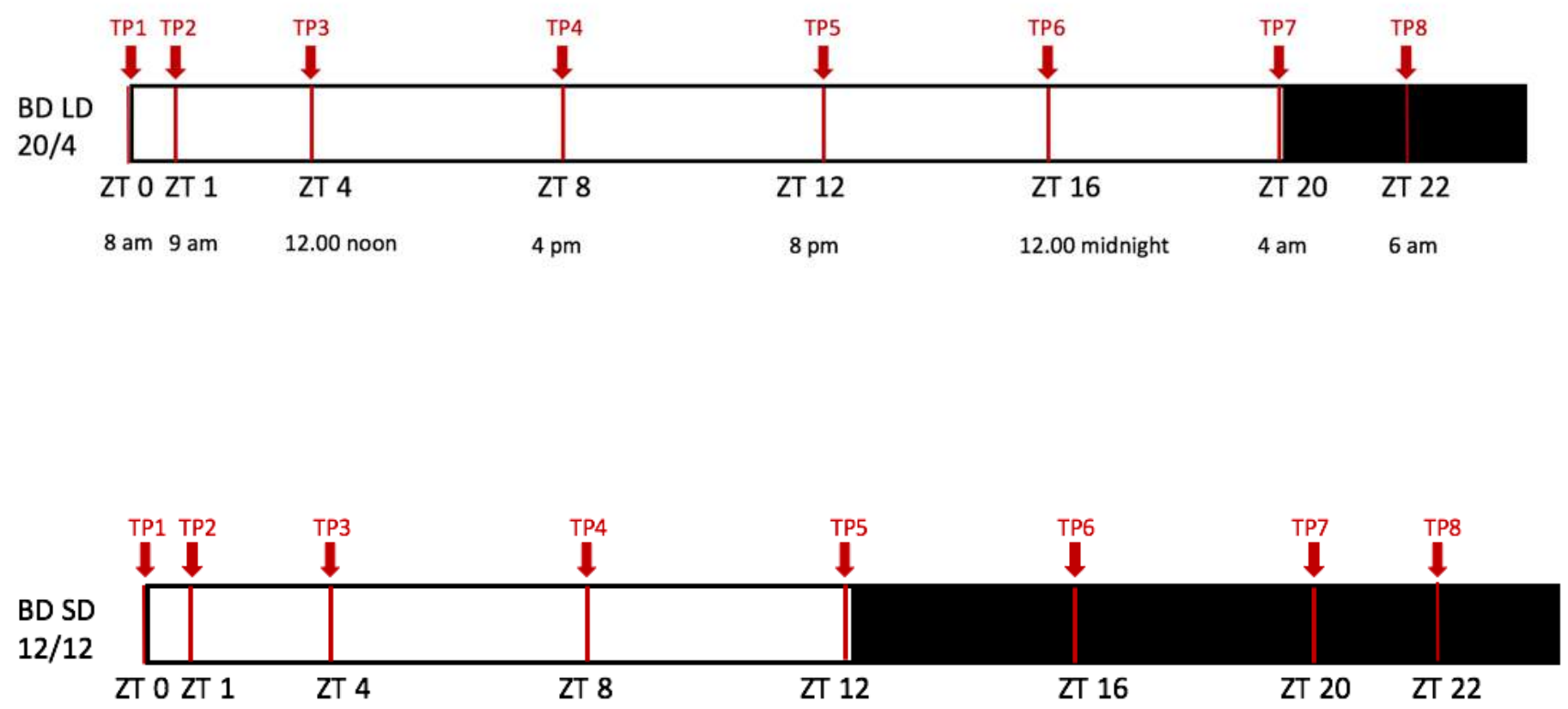
bioRxiv preprint doi: https://doi.org/10.1101/697169; this version posted July 9, 2019. The copyright holder for this preprint (which was not

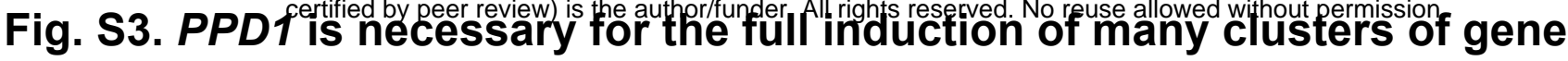
expression that respond to photoperiod. Clustering of RNA-seq time-course data for ppd1-1 plants grown in LD compared to WT. Multiple clusters including 4, 8, 13, 19,22 and 26 show reduced expression in ppd1-1 compared to Bd21-3. Genes which are upregulated are shown in green, genes which are downregulated are shown in red and unchanged is depicted in white.

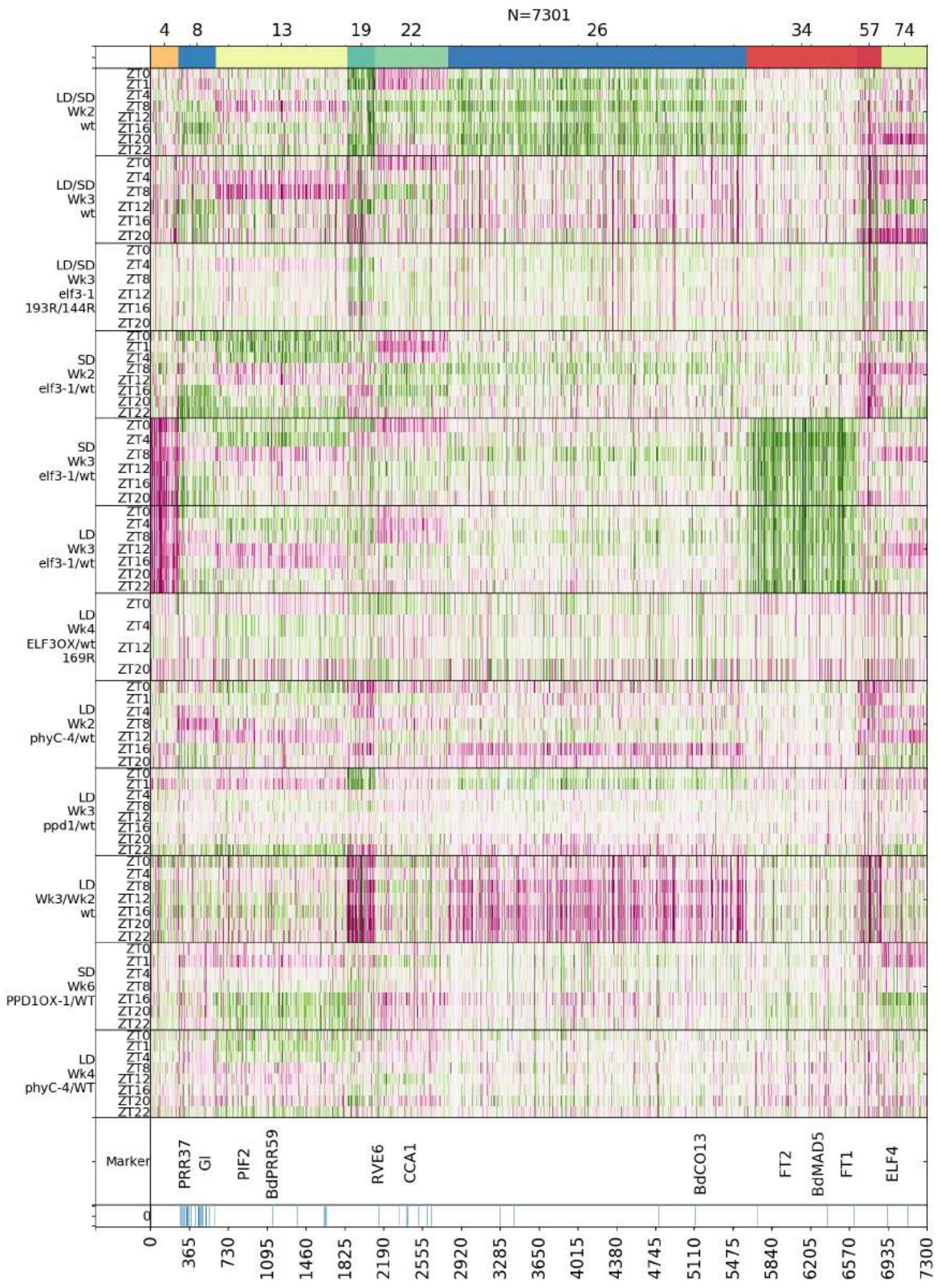


bioRxiv preprint doi: https://doi.org/10.1101/697169; this version posted July 9, 2019. The copyright holder for this preprint (which was not

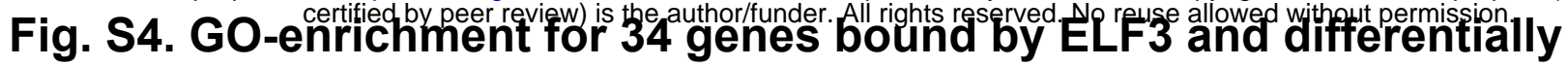
expressed in elf3-1 We identified genes which are up-regulated significantly in elf3-1 compared to wild-type in SD in our transcriptome time-courses. Of these 1169 transcripts, we identified 34 that are directly bound by ELF3. This set of 34 genes that are both bound by ELF3 and whose transcription is regulated by ELF3 is enriched for genes involved in the regulation of flower development, reproductive shoot development as well as response to light, as has been observed in Arabidopsis (gene list in "Datasets", DatasetS5).

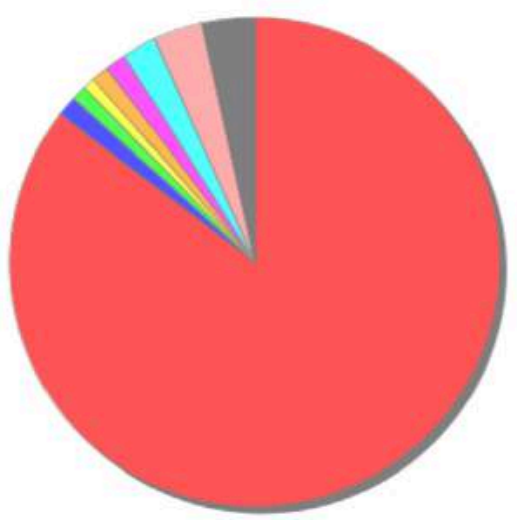

All genes: Brachypodium distachyon

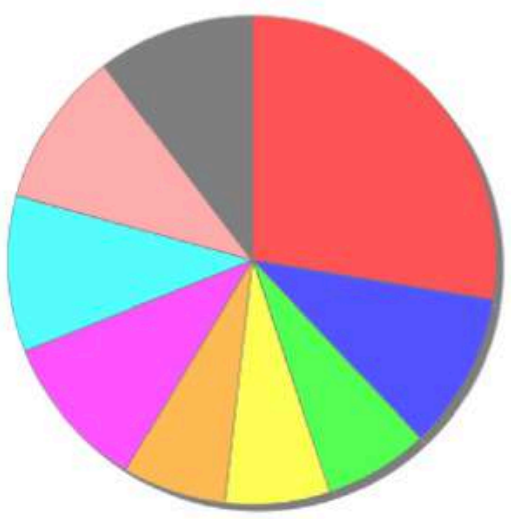

Genes bound by ELF3 in ChIP-seq

biological regulation(GO:0065007)

- flower development(GO:0009908)

post-embryonic development(GO:0009791)

regulation of flower development(GO:0009909)

regulation of shoot system development(GO:0048831)

reproductive shoot system development(GO:0090567)

reproductive structure development(GO:0048608)

response to light stimulus(GO:0009416)

response to radiation(GO:0009314) 
bioRxiv preprint doi: https://doi.org/10.1101/697169; this version posted July 9, 2019. The copyright holder for this preprint (which was not

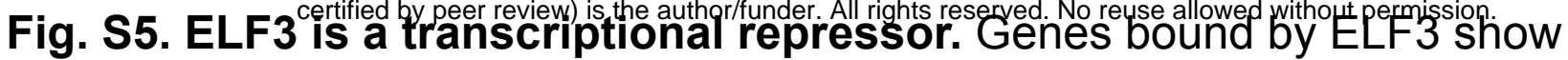
repression at the end of day and during the night. In the absence of ELF3 activity, these genes are no longer repressed during the night but are highly expressed. This indicates that the direct binding of ELF3 causes transcriptional repression. $Y$ axis shows average expression in log scale and $\mathrm{x}$-axis shows $\mathrm{ZT}$, with start at ZTO
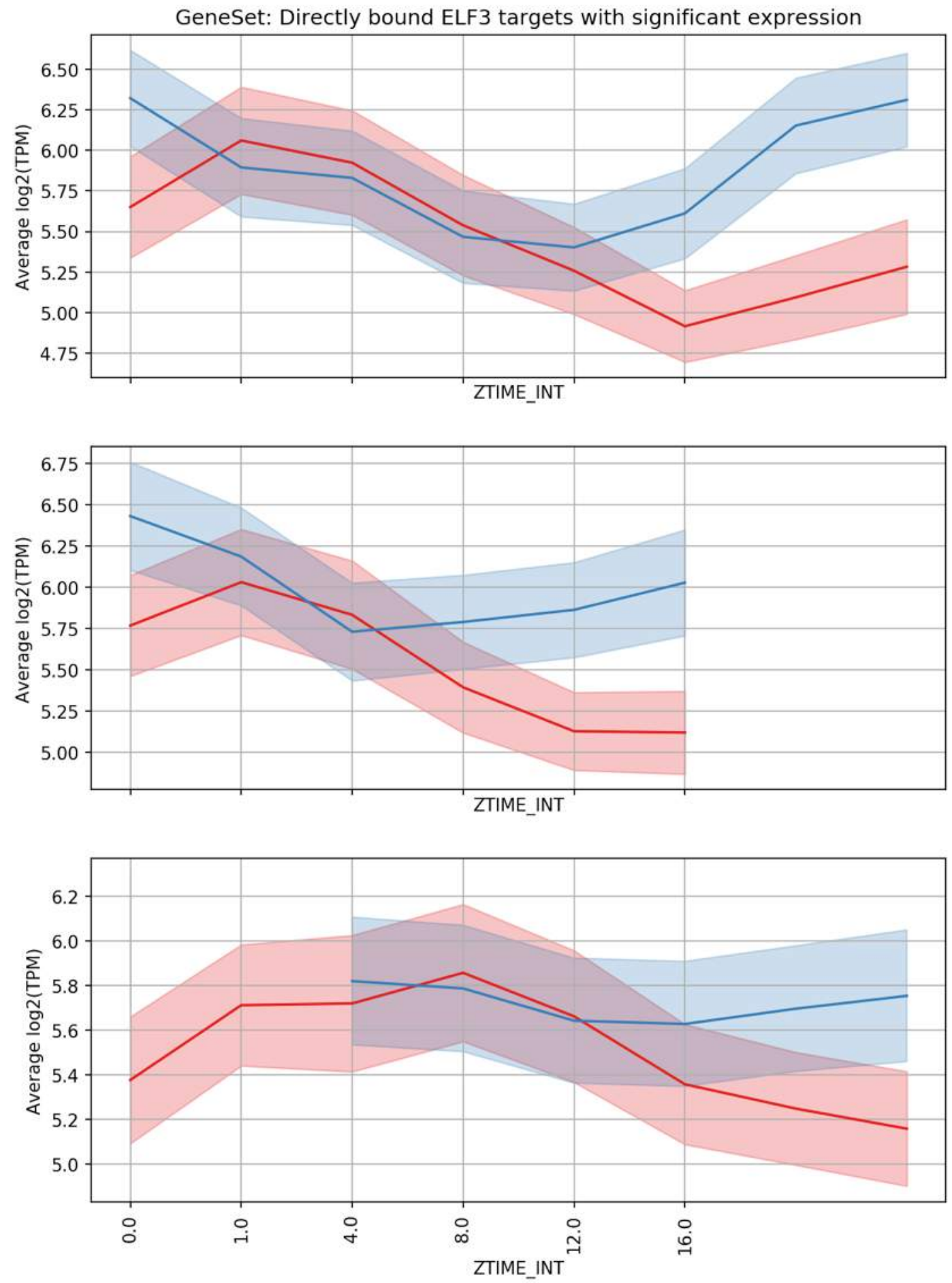
bioRxiv preprint doi: https://doi.org/10.1101/697169; this version posted July 9, 2019. The copyright holder for this preprint (which was not

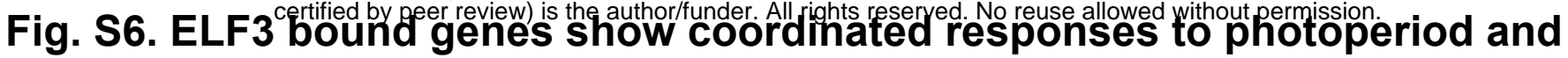
genetic perturbation. Many of the genes that are directly bound by ELF3 are strongly induced by long photoperiods and in elf3-1 (green). These genes are transcriptionally repressed in phyC-4 in LD compared to WT in week 2. PRR37/ $P P D 1$ (red box) shows a particularly strong response to photoperiod, elf3-1 and phyC-4. Perturbing PRR37/PPD1 does not have as large a global effect on the transcriptome as for ELF3 and $P H Y C$, suggesting it is downstream in the pathway.

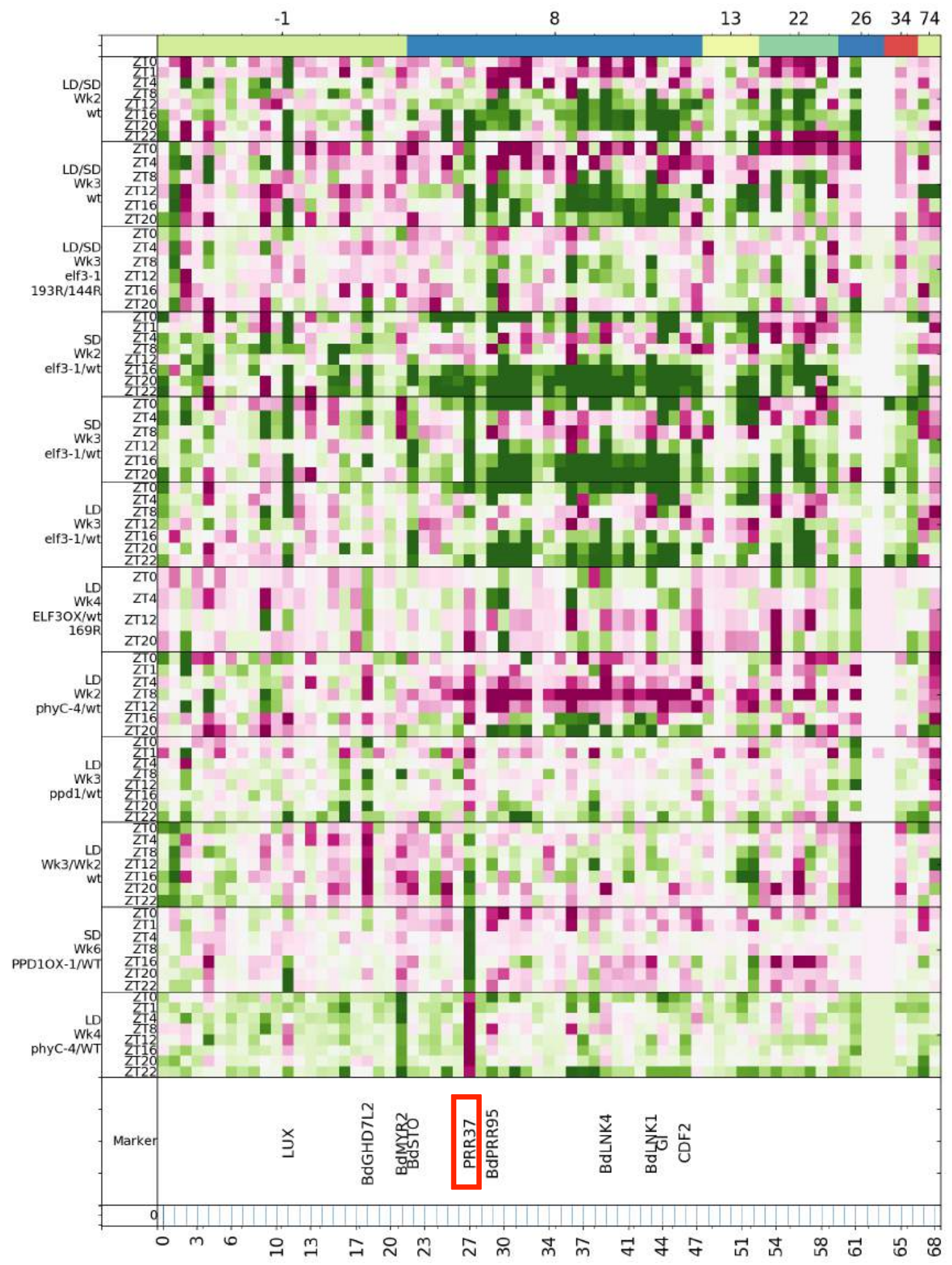


bioRxiv preprint doi: https://doi.org/10.1101/697169; this version posted July 9, 2019. The copyright holder for this preprint (which was not

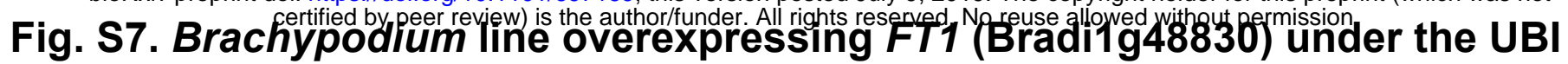
promoter (pUBI) flower almost independently of photoperiod $(A)$ FT1-OX under non-inductive SD conditions (12L:12D), Left: Bd21-3; Right: $p U B I: F T 1$, Bar $=5 \mathrm{~cm}$ (B) FT1-OX plants flower nearly as early under non inductive short day conditions as under long days, whereas wild-type plants do not flower in 12L:12D.
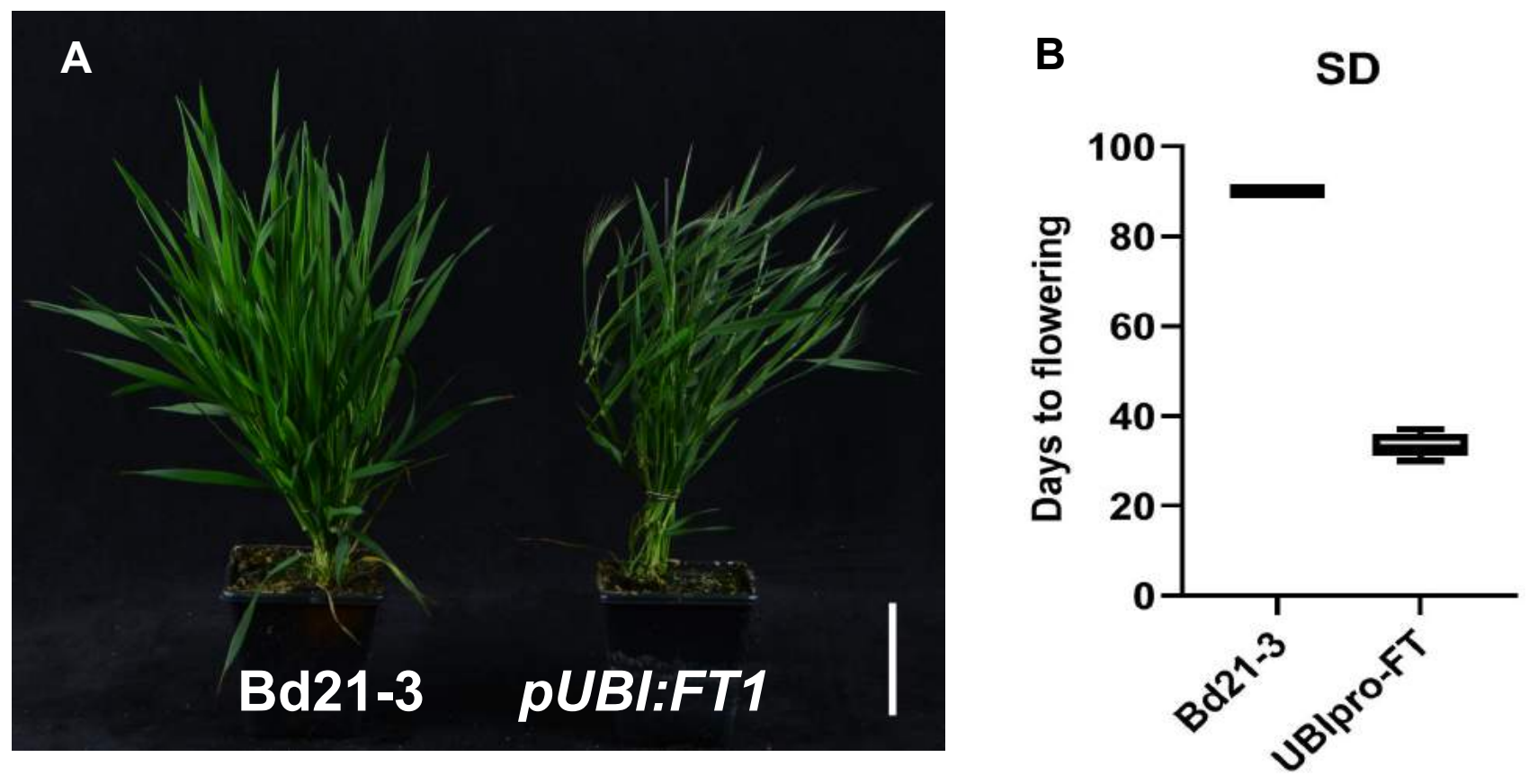
bioRxiv preprint doi: https://doi.org/10.1101/697169; this version posted July 9, 2019. The copyright holder for this preprint (which was not

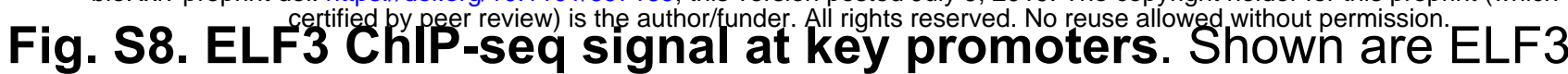
ChIP-seq on the promoters of FT2 and AP1 at ZT0, ZT4 and ZT20 (SD, $12: 12$ and LD (20:4). 3 plants were used per sample. Last row shows the Input control. Height of tracks are shown as enrichment (Reads Per Kilobase Million, RPKM) in IGV browser.
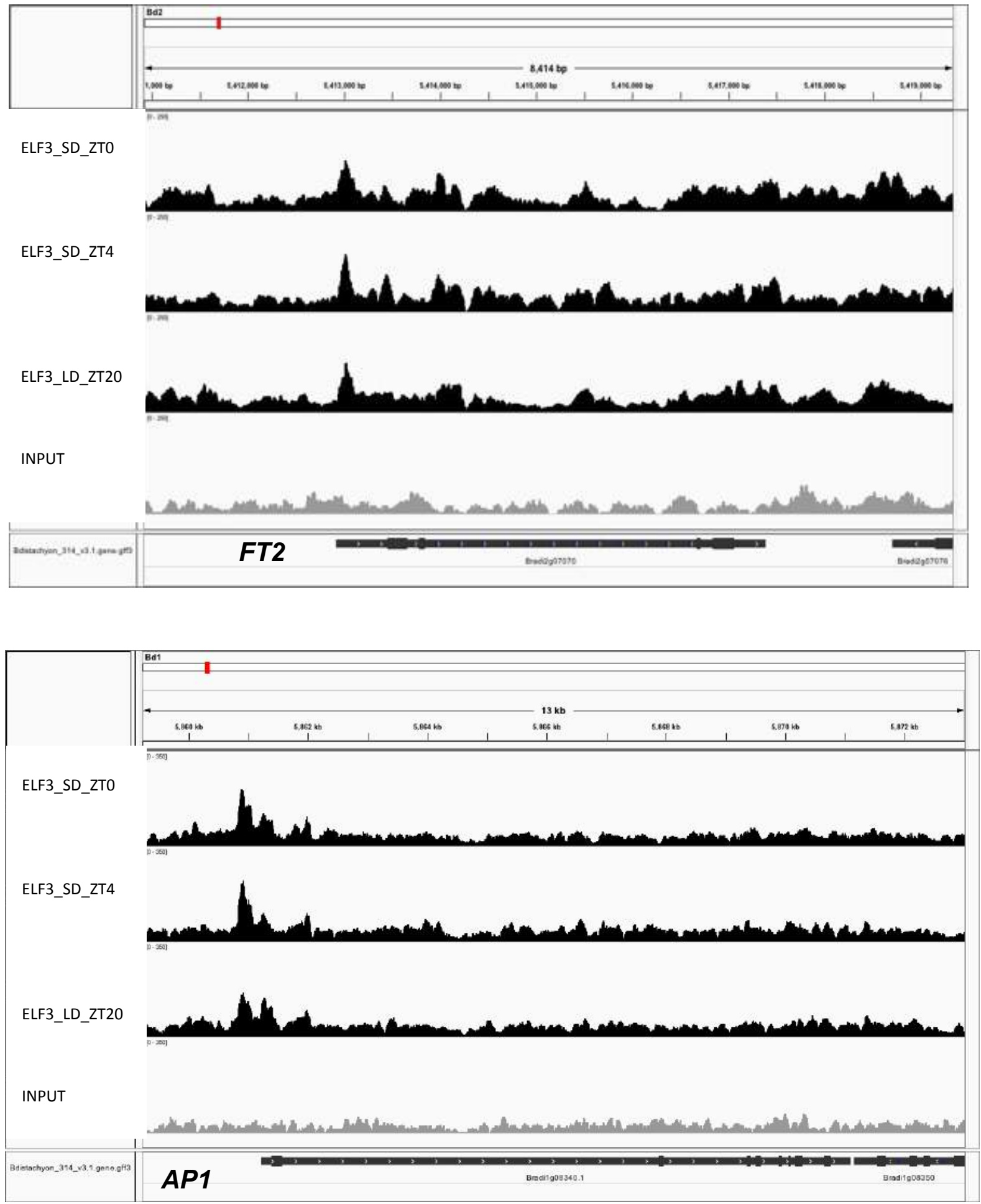

ELF3 ChIP-seq as Signal Reads Per Kilobase Million (RPKM) 
bioRxiv preprint doi: https://doi.org/10.1101/697169; this version posted July 9, 2019. The copyright holder for this preprint (which was not

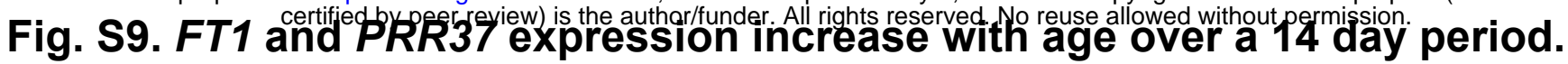

Plants were grown in LD and samples were collected every 2 days at ZT4 starting at 14 DAG, with 3 plants per sample. Expression is shown as transcripts per million (TPM). By comparison, PHYC and ELF3 expression remains largely constant with age.

FT1 expression increases with age

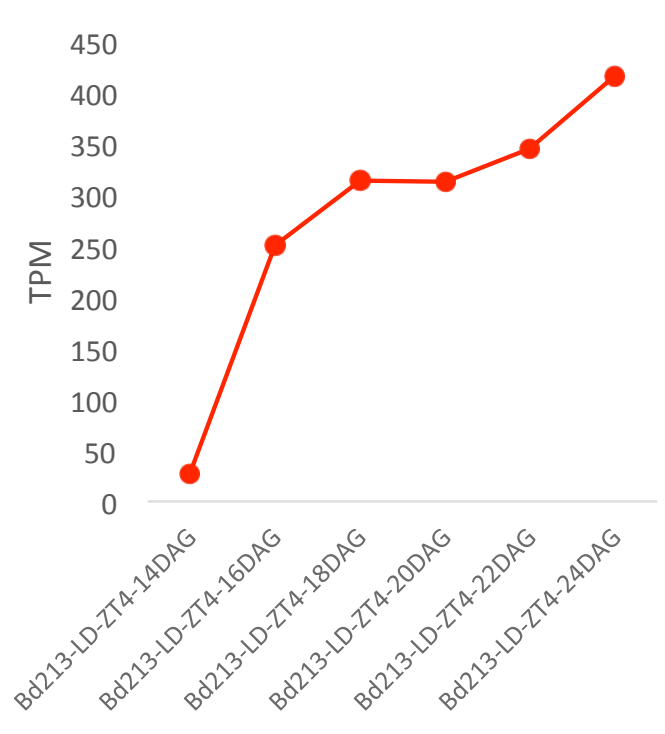

PHYC expression does not increase with age

60

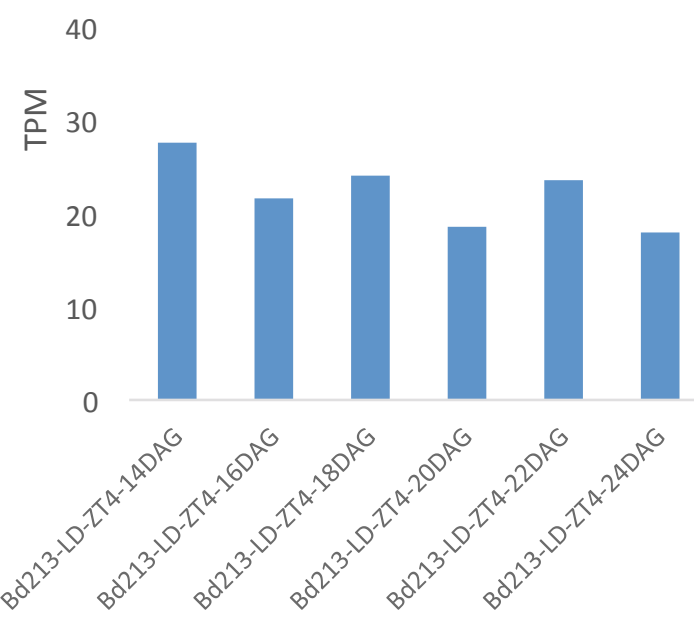

PRR37 expression increases with age up to the floral transition

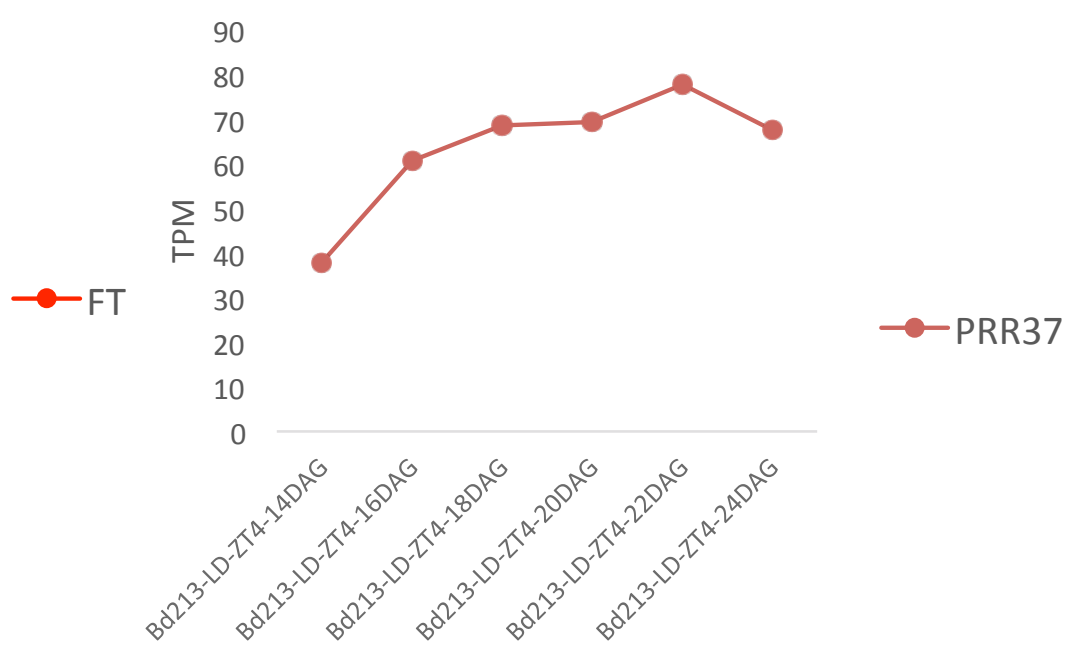

ELF3 expression does not decrease with age

$$
100
$$

90

80

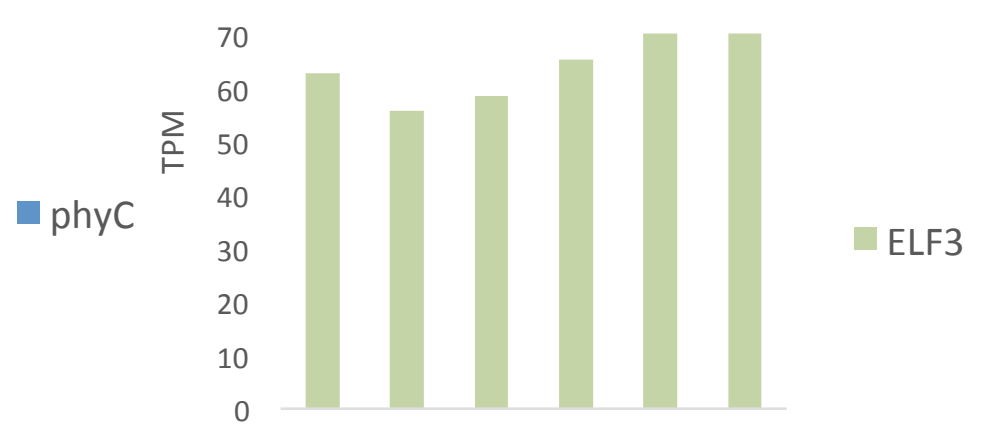


bioRxiv preprint doi: https://doi.org/10.1101/697169; this version posted July 9, 2019. The copyright holder for this preprint (which was not

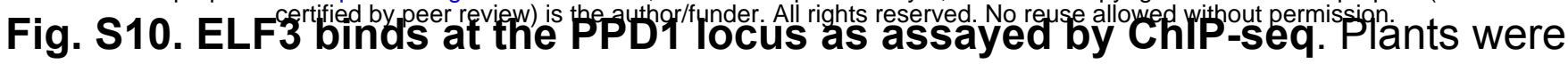
grown (top to bottom) under SD, LD or LL conditions and sampled at ZT0 12 DAG, with 3 plants being used per ChIP-seq sample. Last row shows the Input control. Height of tracks are shown as enrichment (RPKM) in IGV browser. ELF3 binding is reduced in LL compared to SD. ELF3 binds PPD1 just upstream of the ATG.

Analyses of ppd1 alleles indicate that promoter insertions and deletions have played a major role modulating PPD1 expression, revealing a $95 \mathrm{bp}$ region within the promoter just upstream of the ATG that is conserved between wheat, barley and Brachypodium (Seki et al., 2013; Wilhelm, Turner, \& Laurie, 2009), and it had been hypothesized that a photoperiod dependent repressor may bind this 95 bp region in short days to inhibit flowering.

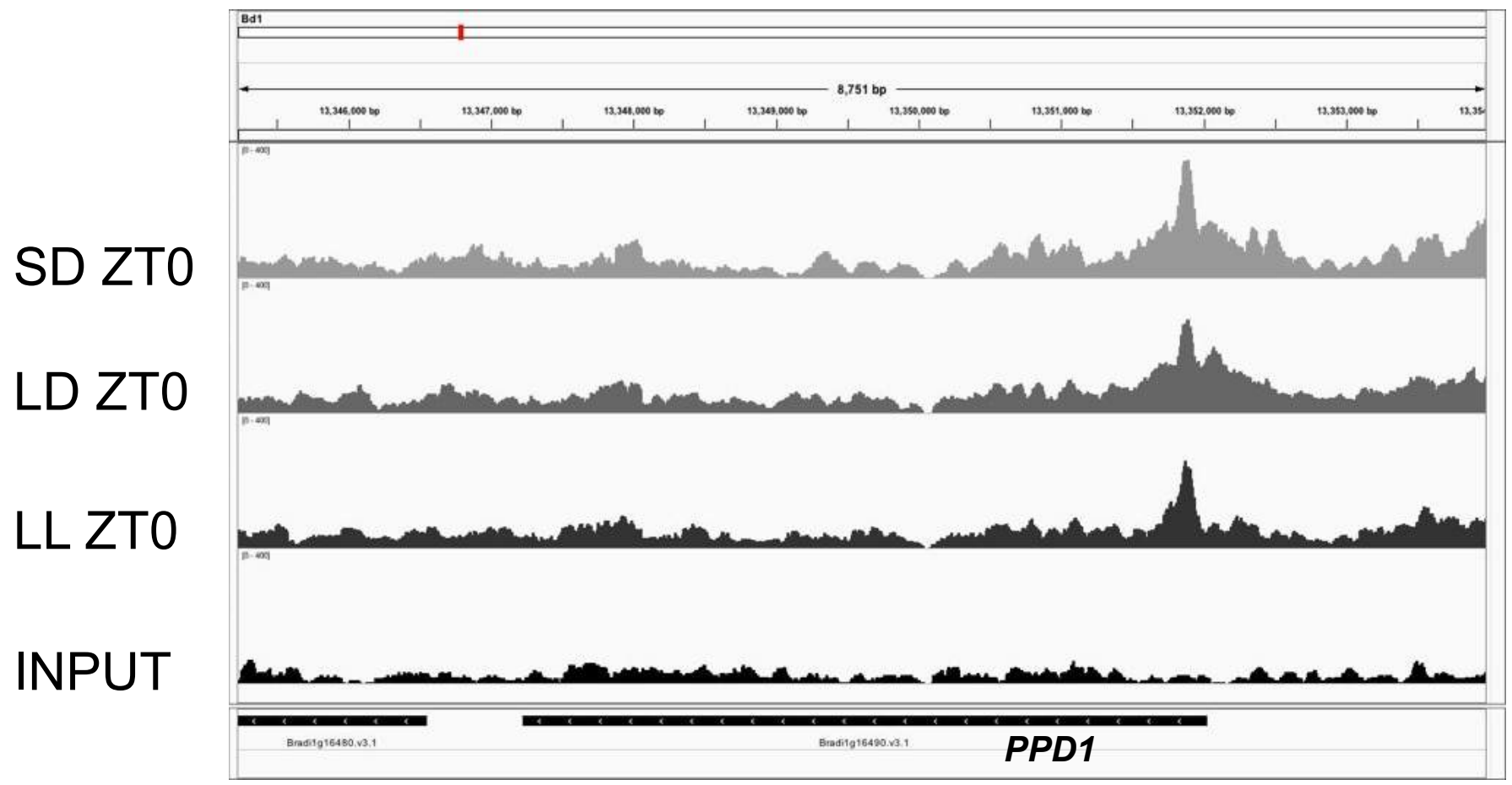

ELF3 ChIP-seq as Signal Reads Per Kilobase Million (RPKM) 
bioRxiv preprint doi: https://doi.org/10.1101/697169; this version posted July 9, 2019. The copyright holder for this preprint (which was not

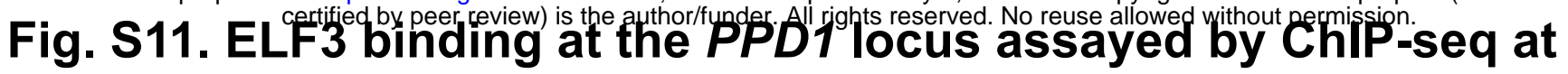
ZT0, ZT4 and ZT20 in SD. Plants were grown in SD (12L:12D) and sampled 12 DAG, with 3 plants per sample. Last row shows Input control. ELF3 shows more binding during the night (ZT0 and ZT20) on PPD1 promoter. Height of tracks are shown as Reads Per Kilobase Million (RPKM) in IGV browser.

\section{ELF3 ChIP-seq Signal}

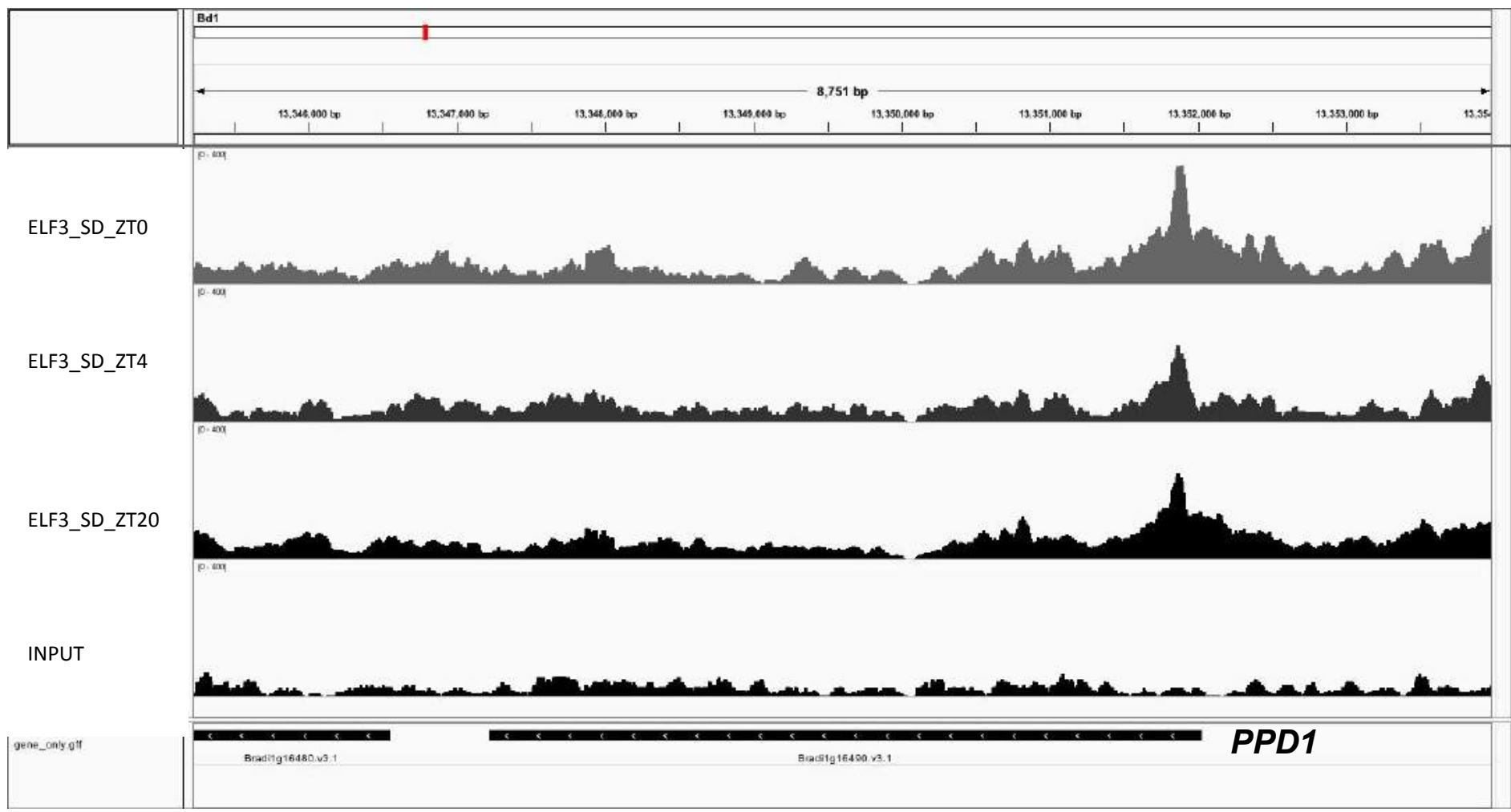

ELF3 ChIP-seq as Signal Reads Per Kilobase Million (RPKM) 
Fig. S12. ELF3 overexpression (ELF3-OX) represses downstream target gene expression. Expression levels of ELF3 bound genes (PPD1, Bradi1g16490; FT2, Bradi2g07070), and a downstream target (FT1, Bradi1g48830) are repressed in an ELF3 overexpression line over a $24 \mathrm{~h}$ time course. Plants were grown under long day conditions and samples collected 3 weeks after germination at the indicated time. Values are given as transcript per million (TPM).
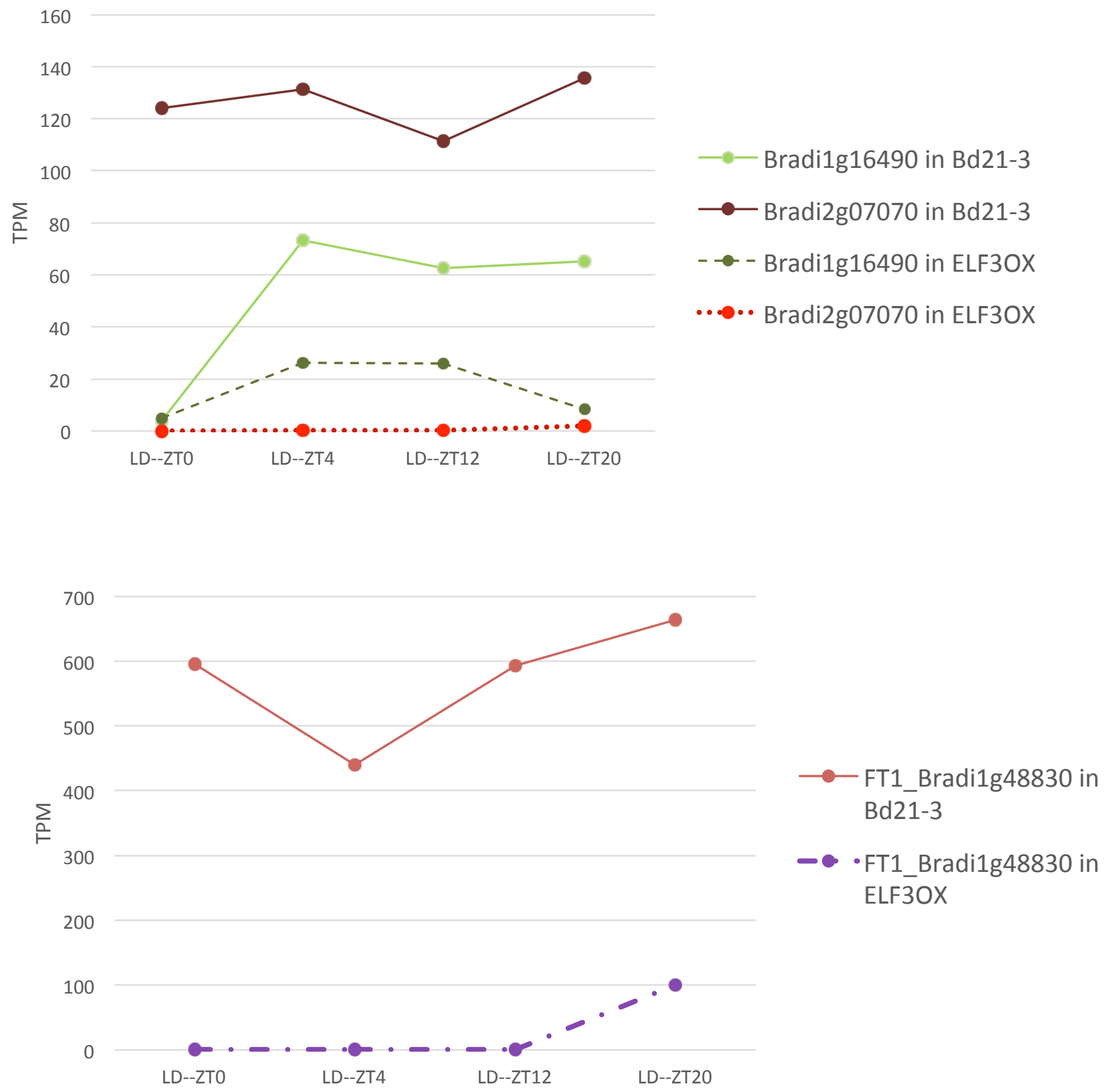
Fig. S13. Expression levels of marker genes in the pUBI:PPD1-Flag line and ChIP-seq of PPD1. FT1 (Bradi1g48830), FT2 (Bradi2g07070) and AP1

(Bradi1g08340) are highly upregulated in a PPD1-OX line under non-inductive SD conditions (12L:12D). Plants were grown for 6 weeks under SD condition and sampled at the indicated time, 3 leaves from individual plants were mixed for each sample. RNA-seq results shown as transcript per million (TPM).

PPD1 binds directly to 5' region of FT2 and AP1 as assayed by ChIP-seq. Top row (black) shows PPD1-OX-Flag ChIP-seq, and grey row shows Input control. Displayed in IGV browser.
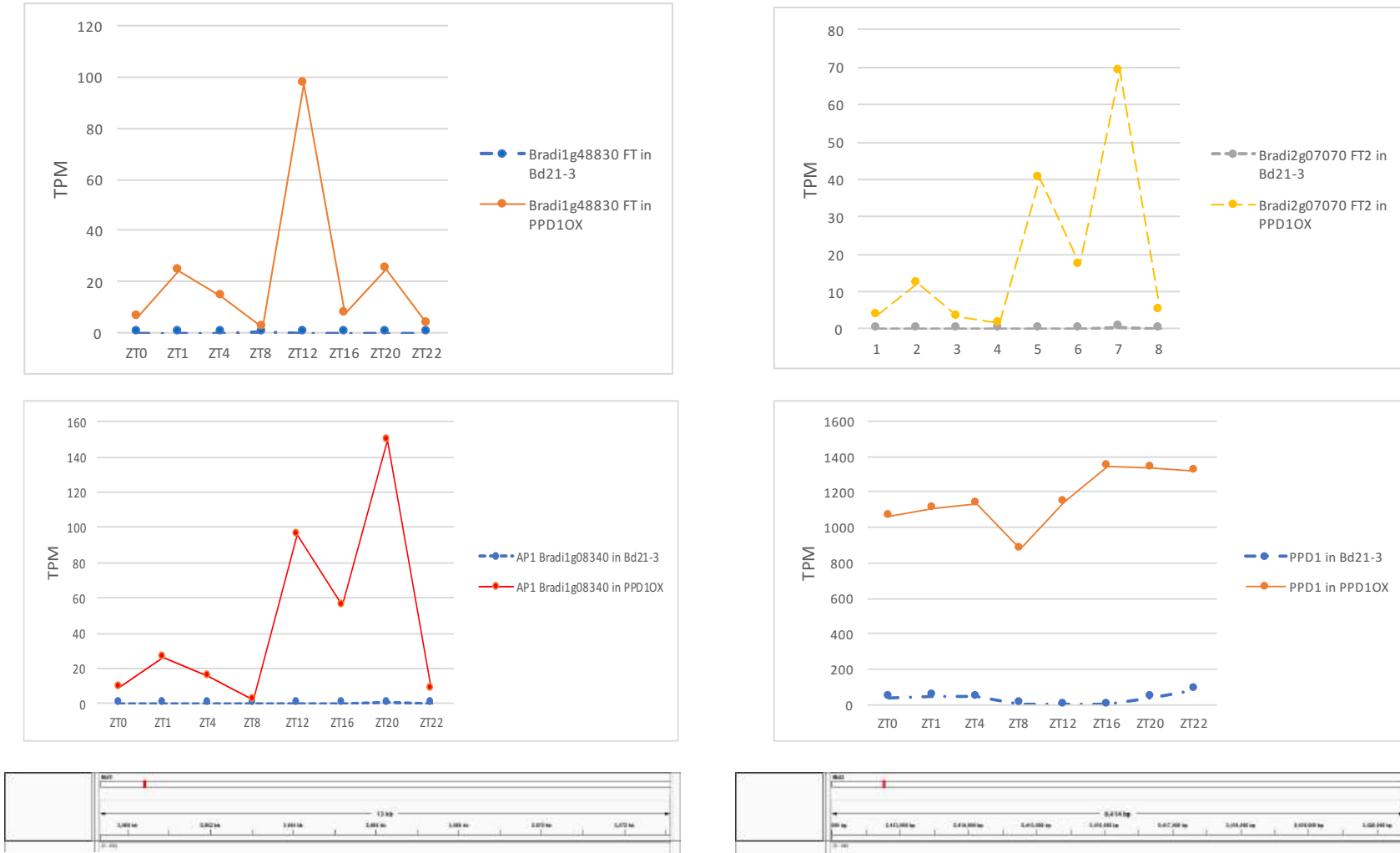

PPD1
LD_ZT4

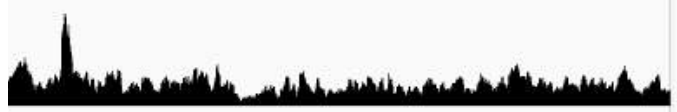

INPUT
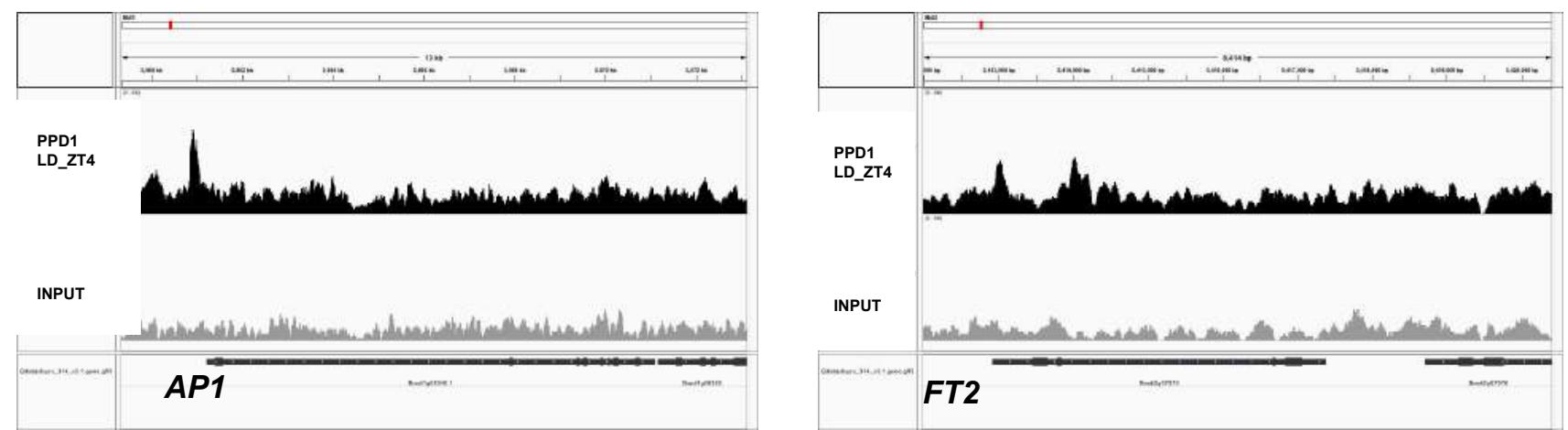

PPD1 ChIP-seq as Signal Reads Per Kilobase Million (RPKM) 
Fig. S14. ELF3 expression is not affected in phyC-4, but FT1 expression is abolished. Plants were grown under long day conditions (20L:4D) and samples collected 4 weeks after germination at the indicated time. A. ELF3 expression. B. FT1 expression. Values are given as transcript per million (TPM).

A

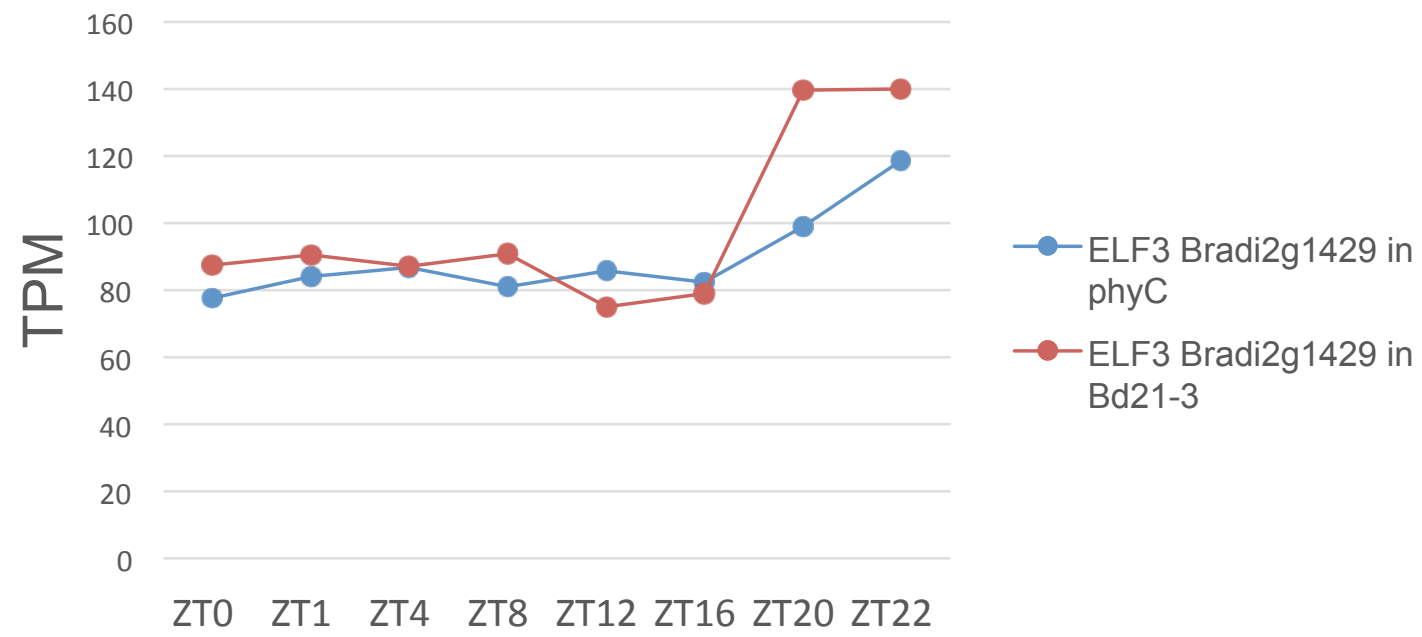

B

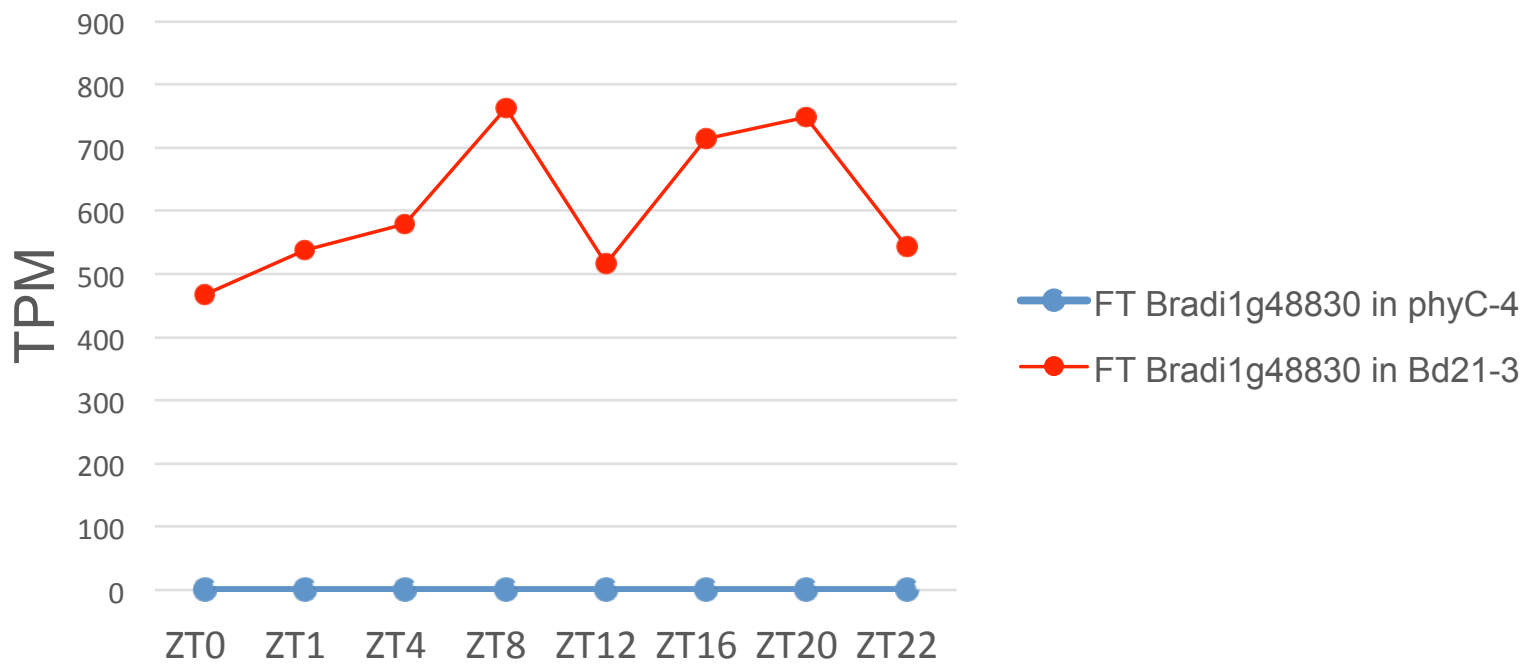


Fig. S15. ELF3 protein is degraded in response to light. Additional Western blots showing degradation of ELF3 in response to light with antibody against ELF3 under SD (12:12) or 20L:12D condition. Plants were grown under 12L:12D (SD) or 20L:12D condition as indicated and samples taken 12 DAG at the indicated time (ZT20, ZT0 and ZT4, with 3 plants used per sample). We used wild-type plants (lane 2) or plants overexpressing ELF3 ( $p$ UBI:ELF3-GFP-Flag) (lane 3 to lane 7) and probed with an antibody raised in rabbit against ELF3 peptide (Agrisera).

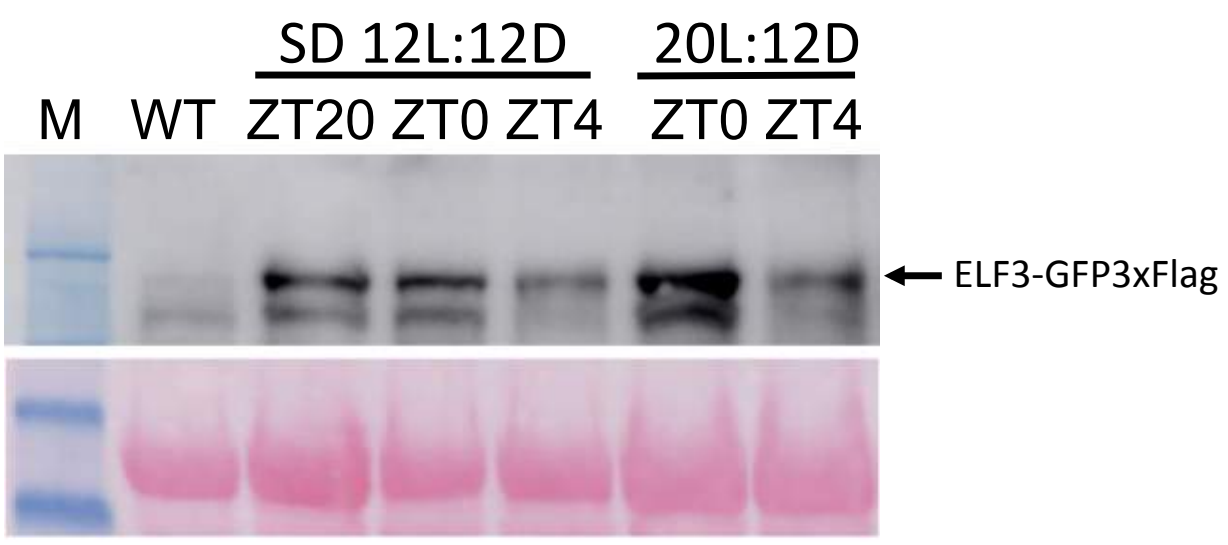


Fig. S16. Enrichment of ELF3 and phyC at the PPD1 promoter. Plants were grown (top to bottom) under LD, SD or LL conditions and sampled at ZT0 12 DAG (ELF3) or ZT0 and ZT20, LD (phyC), with 3 plants being used per ChIP sample. Last row shows the INPUT control. Height of tracks are shown as enrichment (RPKM) in IGV browser.

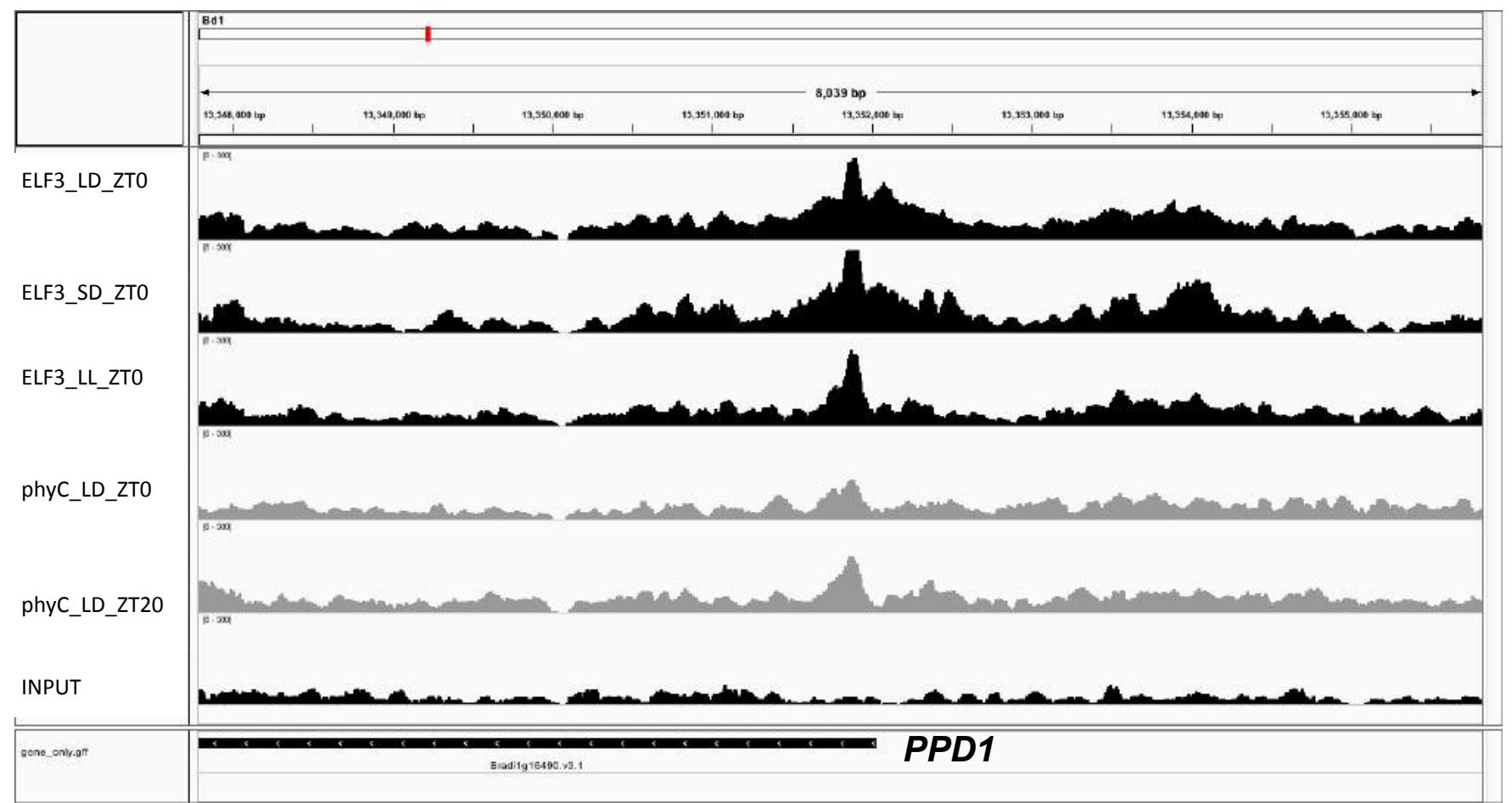

ELF3 and phyC ChIP-seq as Signal Reads Per Kilobase Million (RPKM) 
Fig. S17. Night length, but not day length, determines flowering in Brachypodium. A. Plants grown under $12 \mathrm{~h}$ light and $4 \mathrm{~h}$ dark (12L: 4D) condition flower nearly at the same time as plants grown in 20L:4D (LD) conditions, whereas plants grown under 12L:12D, 20L:12D or 12L: 20D did not flower during the course of the experiment. Experiment was terminated at 75 days, when plants began to senesce. Brachypodium does not flower when grown under long night conditions, regardless of day length. B. From left to right: Bd21 grown under 20L:4D (LD), 12L: 20D and 20L:12D. C. Brachypodium flowers early when grown under short night conditions, regardless of day length. From the left to right: $B d 21$ grown under 20L:4D (LD), 12L:12D (SD) or 12L:4D

A.
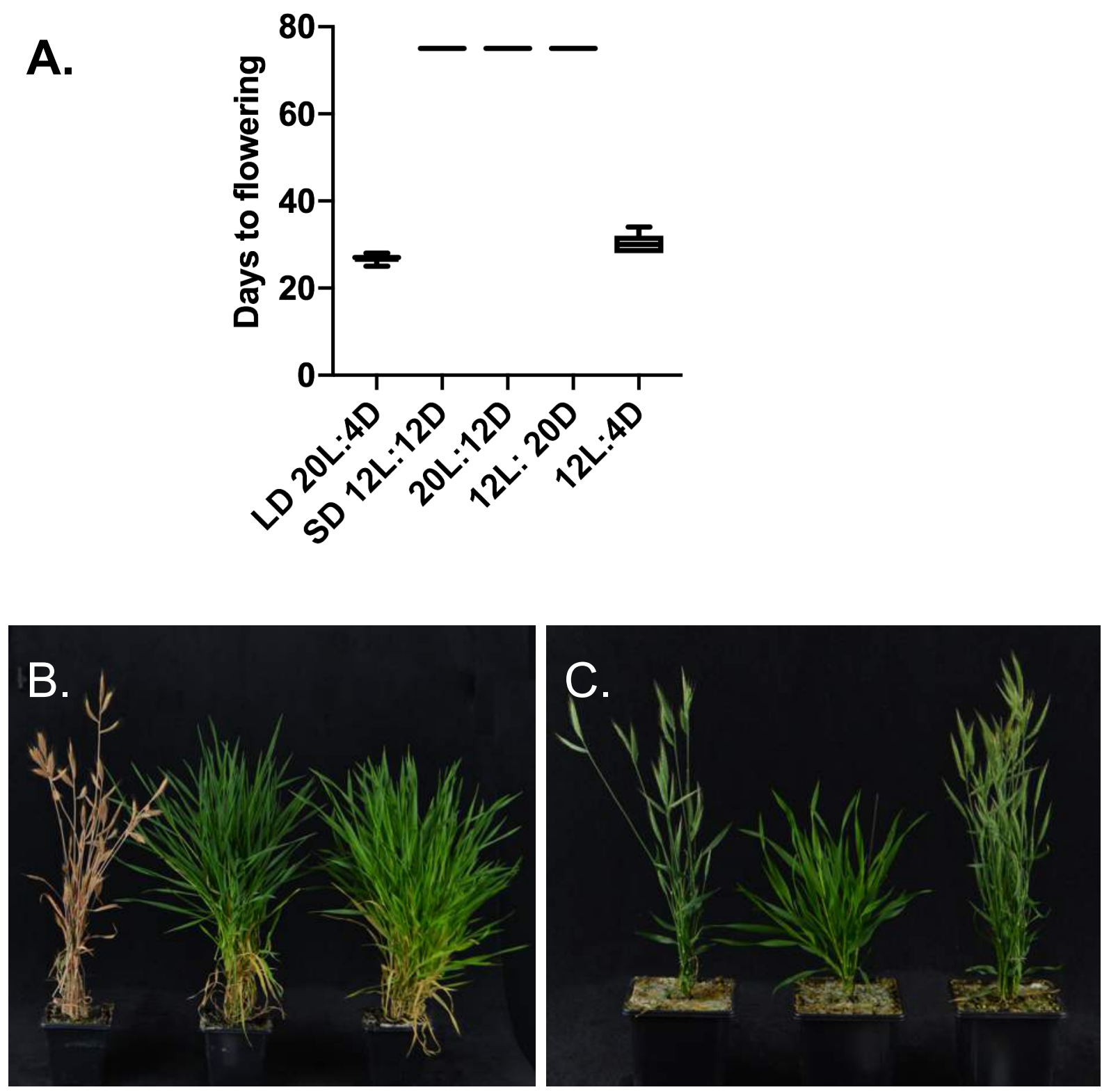
Fig. S18. A night break is sufficient to trigger flowering under non-inductive short day conditions in Brachypodium. Introducing a single $1 \mathrm{~h}$ night break or 2 night breaks of 30 min results in a flowering time similar to a plant being grown under inductive long days. Conditions:

2*1h NB: 12 hour light +4 hours dark +1 hour light +3 hours dark +1 hour light +3 hours dark.

$2 * 0.5$ h NB: 12 hour light +4 hours dark +0.5 hour light +3.5 hours dark +0.5 hour light +3.5 hours dark.

1*1h NB: 12 hour light +6 hours dark +1 hour light +5 hours dark, all Bd21

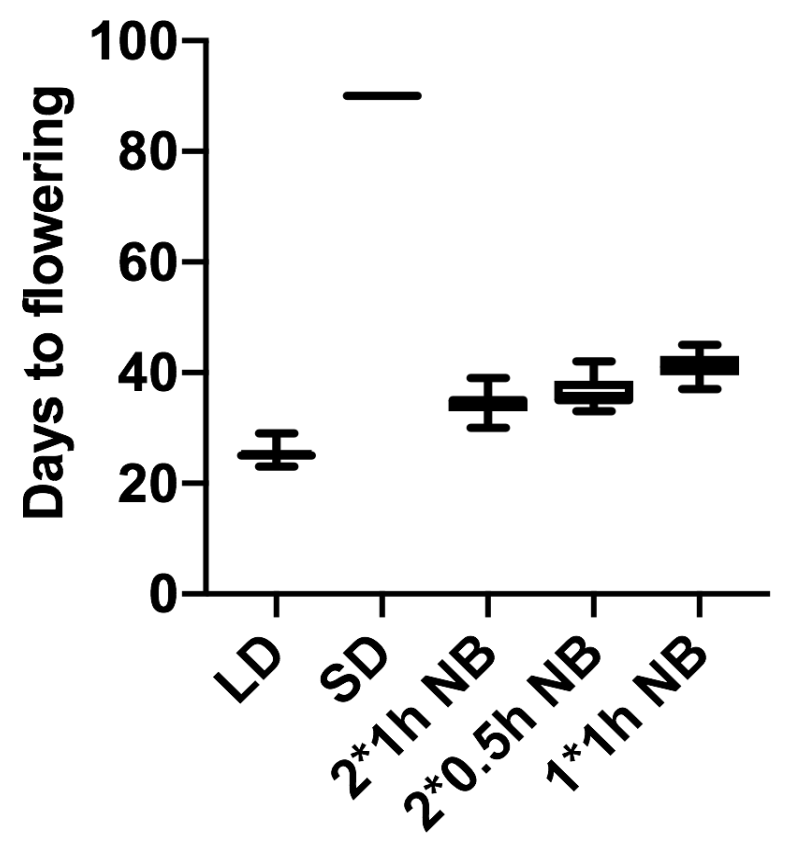

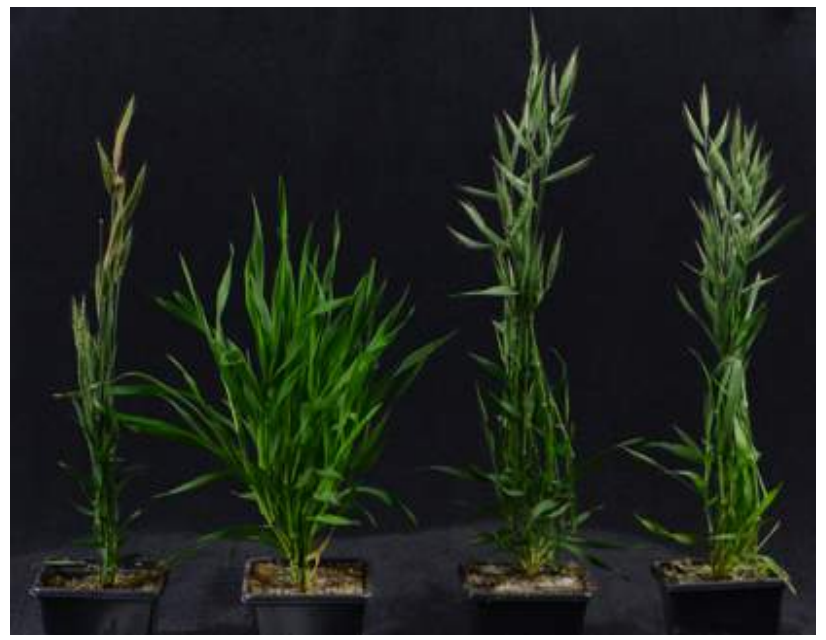

LD SD $\quad 2 * 1 \mathrm{~h} \mathrm{NB} \quad 2 * 0.5 \mathrm{~h} \mathrm{NB}$

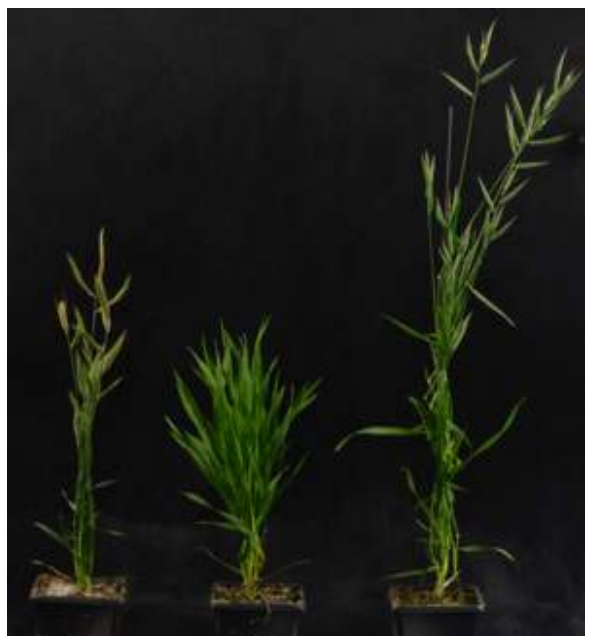

LD SD $\quad 1 * 1 \mathrm{~h} \mathrm{NB}$ 
Fig. S19. Detection of key proteins by immunoblot in plant extracts. A. phyC and PRR37 protein levels are stable and do not change at different times of day. Plants were grown under 20L:4D (LD) condition and samples taken 12 DAG at the indicated time (ZT20, ZT0 with 3 plants used per sample). We used 2 independent lines expressing $p U B I$ :phyCGFP-Flag and 1 expressing $p U B I: P R R 37-F l a g$ probed with an antibody against Flag epitope (M2, Sigma). B. 35S-NFlag-BdPHYC is stably expressed in phyABCDE Arabidopsis. We tested 6 independent lines for expression level of BdphyC. Western blot was probed with with an antibody against Flag epitope (M2, Sigma).

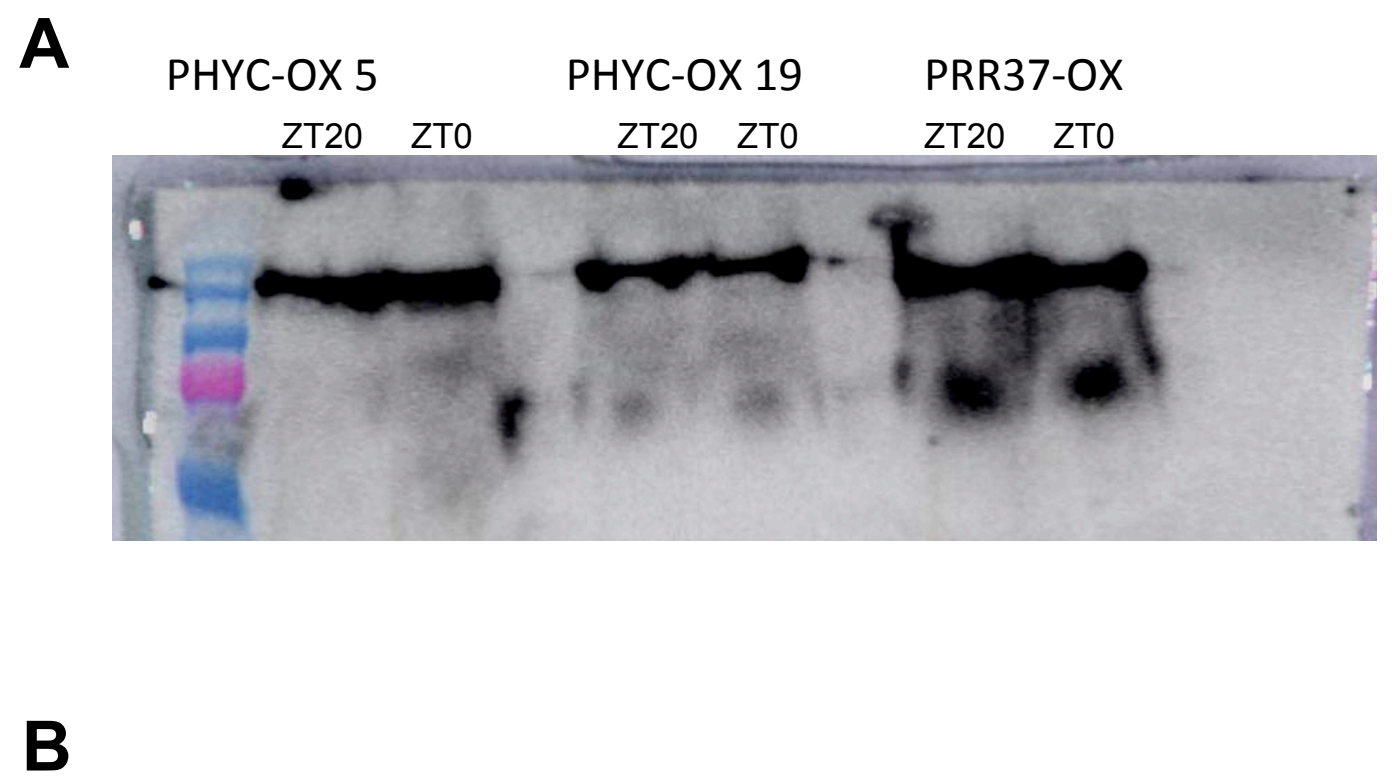

35S-NFLAG-BdPHYC in phyABCDE

$\begin{array}{llllllll}M & \text { Col } & 1 & 2 & 3 & 4 & 5 & 6\end{array}$

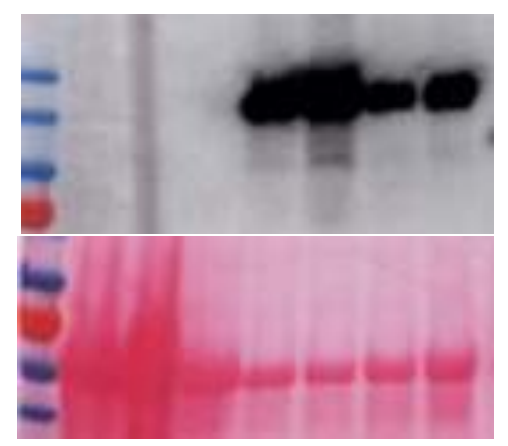


Fig S20. The dark reversion of phyC Pfr is suitable to provide a measure of the length of the night. During SD, PhyC Pfr declines to low levels during the longer nights, enabling greater accumulation of the floral repressor ELF3. In LD, nights are short and phyC Pfr levels remain high. This prevents ELF3 accumulation, allowing higher levels of $P P D 1$ to be expressed, allowing the activation of $F T$.

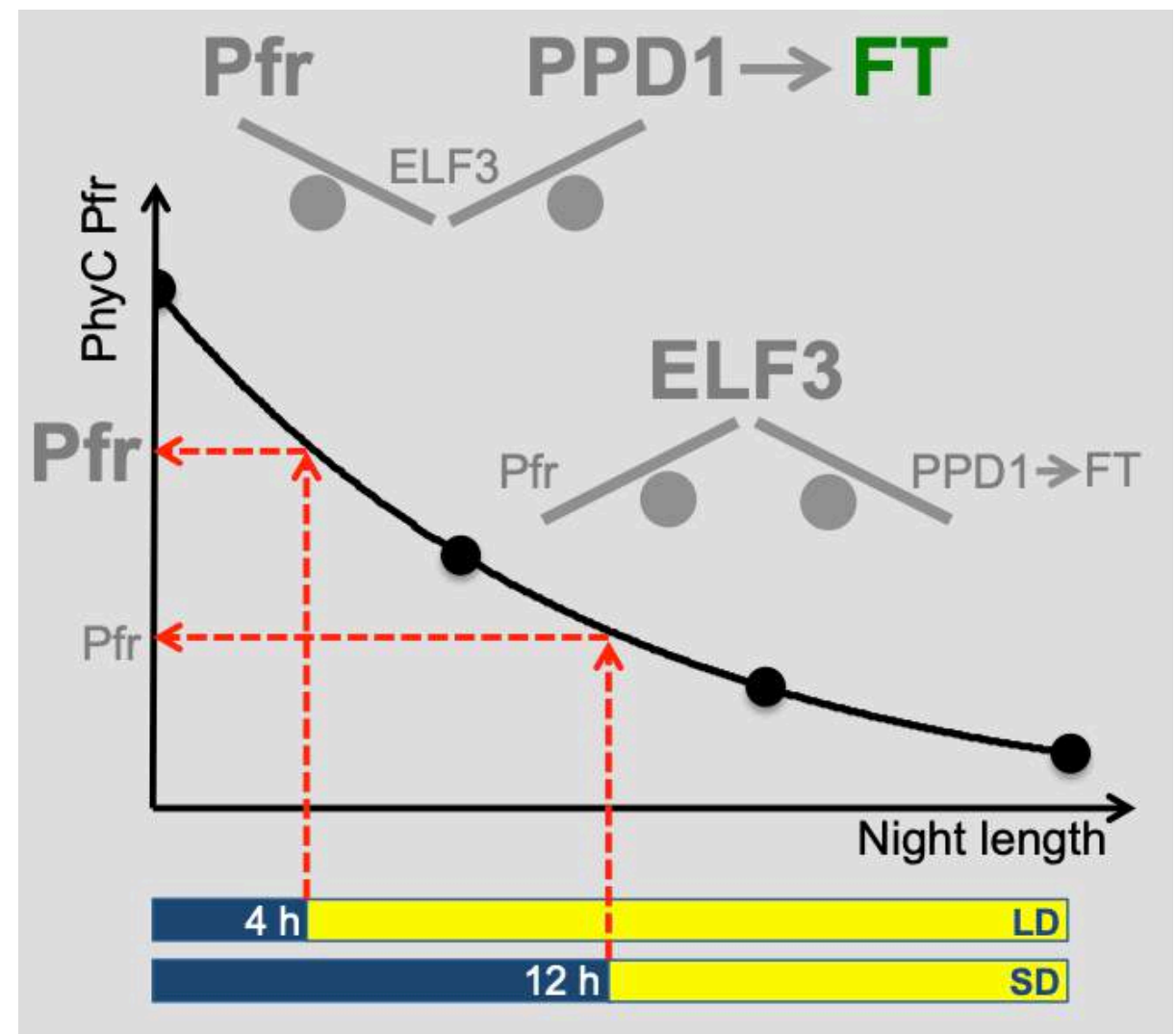




\title{
Supplementary Materials for: Phytochromes measure photoperiod in Brachypodium
}

\author{
Authors: \\ Mingjun Gao ${ }^{1}$, Feng Geng ${ }^{1}$, Cornelia Klose ${ }^{2}$, Anne-Marie Staudt ${ }^{2}$, He Huang ${ }^{3}$, Duy \\ Nguyen $^{1}$, Hui Lan ${ }^{1}$, Todd C. Mockler ${ }^{3}$, Dmitri A. Nusinow ${ }^{3}$, Andreas Hiltbrunner ${ }^{2,4}$, \\ Eberhard Schäfer $^{2,5}$, Philip A. Wigge ${ }^{1,6,7}$ and Katja E. Jaeger ${ }^{1,6} *$
}

\begin{abstract}
Affiliations:
${ }^{1}$ Sainsbury Laboratory, University of Cambridge, 47 Bateman St., Cambridge CB2 1LR, UK.

${ }^{2}$ Institut für Biologie II, University of Freiburg, Schaenzlestr. 1, D-79104 Freiburg, Germany.

${ }^{3}$ Donald Danforth Plant Science Center, St. Louis, MO 63132, USA

${ }^{4}$ Signalling Research Centres BIOSS and CIBSS, University of Freiburg, Schaenzlestr. 18, 79104 Freiburg, Germany

${ }^{5}$ BIOSS Centre for Biological Signalling Studies, University of Freiburg, Schaenzlestr. 18, 79104 Freiburg, Germany

${ }^{6}$ Leibniz-Institut für Gemüse- und Zierpflanzenbau, Theodor-Echtermeyer-Weg 1, 14979

Großbeeren, Germany

${ }^{7}$ Institute of Biochemistry and Biology, University of Potsdam, 14476 Potsdam, Germany

*Correspondence to: jaeger@igzev.de
\end{abstract}

\section{This PDF file includes:}

Materials and Methods

Figs. S1 to S20

References

Datasets:

Dataset S1: Mass spec results for protein identification

Dataset S2: RNA-seq tables for Bd21-3, elf3-1, phyC-4 and ppd1-1

Dataset S3: Cluster lists for RNA-seq

Dataset S4: Genes bound by ELF in ChIP-seq and perturbed in elf3-1

Dataset S5: ChIP-seq peaks for phyC

Dataset S6: Dark reversion measurements for phyC 


\section{Materials and Methods}

\section{Plant materials and growth conditions}

Brachypodium distachyon accession $B d 21-3$ and $B d 21$ were used in this study. Seeds were imbibed in distilled water at $4{ }^{\circ} \mathrm{C}$ for two days before sowing. Plants were grown in 5 parts John Innes \#2, 3 parts peat, 1 parts silver sand, 3 parts course vermiculite, Osmocote $2.7 \mathrm{~g} / \mathrm{L}$. All plants were grown in growth cabinets with constant temperature $20{ }^{\circ} \mathrm{C}, 65 \%$ humidity and $350 \mu \mathrm{mol} \mathrm{m} \mathrm{m}^{-2}$ PPFD (Photosynthetic Photon Flux Density). For flowering-time experiments, plants were grown in photoperiod regimes: (a) LD (20 h light/4 h dark); (b) SD (12 h light/12 h dark); (c) 20:12 (20 h light/12 h dark); (d) 12:20 (12h light /20 h dark), (e) 12:4 (12 h light/4 h dark).

\section{Mutants used in this study}

\begin{tabular}{|c|c|c|c|c|}
\hline $\begin{array}{l}\text { Line } \\
\text { Name }\end{array}$ & Background & Description & Source & Notes \\
\hline elf3-1 & $B d 21-3$ & crispr line & This study & $\begin{array}{l}\text { 7bp deletion or } 1 \mathrm{bp} \text { insertion } \\
\text { in the second exon, both } \\
\text { caused premature stop codon }\end{array}$ \\
\hline phyc-4 & $\mathrm{Bd} 21-3$ & crispr line & This study & $\begin{array}{l}\text { 4bp deletion in the first exon, } \\
\text { caused premature stop codon }\end{array}$ \\
\hline ppd1-1 & $\mathrm{Bd} 21-3$ & crispr line & This study & $\begin{array}{l}1 \mathrm{bp} \text { deletion in the sixth exon, } \\
\text { caused premature stop codon }\end{array}$ \\
\hline phyA-1 & $\mathrm{Bd} 21-3$ & crispr line & This study & $\begin{array}{l}\text { 3bp deletion in the first exon, } \\
\text { caused one amino acid } \\
\text { deletion }\end{array}$ \\
\hline phyB-1 & $\mathrm{Bd} 21-3$ & T-DNA & $\begin{array}{l}\text { https://jgi.doe.gov/our- } \\
\text { science/science- } \\
\text { programs/plant- } \\
\text { genomics/brachypodium/br } \\
\text { achypodium-t-dna- } \\
\text { collection/ }\end{array}$ & T-DNA insertion in the $5^{\prime}$ UTR. \\
\hline $\begin{array}{l}\text { UBIpro- } \\
\text { ELF3 }\end{array}$ & $\mathrm{Bd} 21-3$ & $\begin{array}{l}\text { transgenic } \\
\text { line }\end{array}$ & This study & \\
\hline $\begin{array}{l}\text { UBIpro- } \\
\text { PPD1 }\end{array}$ & $\mathrm{Bd} 21-3$ & $\begin{array}{l}\text { transgenic } \\
\text { line }\end{array}$ & This study & \\
\hline
\end{tabular}


The phyC-1 EMS mutant has been described previously (1). The Arabidopsis thaliana phytochrome mutants in Ler backgrounds phyabde, and phyabcde were provided by $\mathrm{K}$. Franklin.

For this study we created CRISPR mutation in the ELF3 gene (Bradi2g14290), PHYC gene (Bradilg08400) and PPD1 gene (Bradilg16490). The cloning of the single guide RNA (sgRNA) was done as described in (2). sgRNAs primers for ELF3, PPD1 and PHYC were designed using design tool http://www.e-crisp.org/E-CRISP/. The annealed oligos were ligated into entry vector pOs-sgRNA and then cloned into destination vector pH-Ubi-cas9-7 by gateway LR reaction. The constructs were transformed in the Agrobacterium strain AGL1. Agrobacterium-mediated plant transformation of embryonic callus generated from immature embryos was performed as described (3). For the genotyping analysis, mutations were confirmed by sequencing and T2 lines with mutation but not carrying Hyg resistance and were selected for further analysis.

For the overexpressing transgenic lines, the genomic coding sequence of ELF3, PPD1 and PHYC were amplified by PCR with primers indicated in Table S1. The PCR products were cloned into SLIC binary vector containing Ubiquitin promoter and C-terminal 3xFLAG tag using NEBuilder® HiFi DNA Assembly Cloning Kit (E2621L). pENTR-

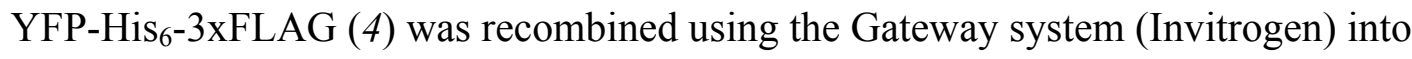
pMDC32 (5). Embryogenic calli from B. distachyon 21-3 plants were transformed with pENTR-YFP-His $6_{6}-3 x$ FLAG as described (6). For each construct, approximately 20 independent transgenic lines were obtained and homozygous single insertion lines were selected for further analysis.

For overexpression of $P H Y C$ in Arabidopsis, the $P H Y C$ genomic fragment was amplified and then cloned into $35 \mathrm{~S}$ and N-terminal 3xFLAG tagged binary vector by NEBuilder ${ }^{\circledR}$ HiFi DNA Assembly Cloning Kit (E2621L). The binary construct was transformed into Arabidopsis phyABCDE mutant by floral dipping method. The 35S-N3FLAG-PHYC transgenic plants were isolated by Kanamycin selection and propagated to obtain homozygous seeds to measure the dark reversion rate. 


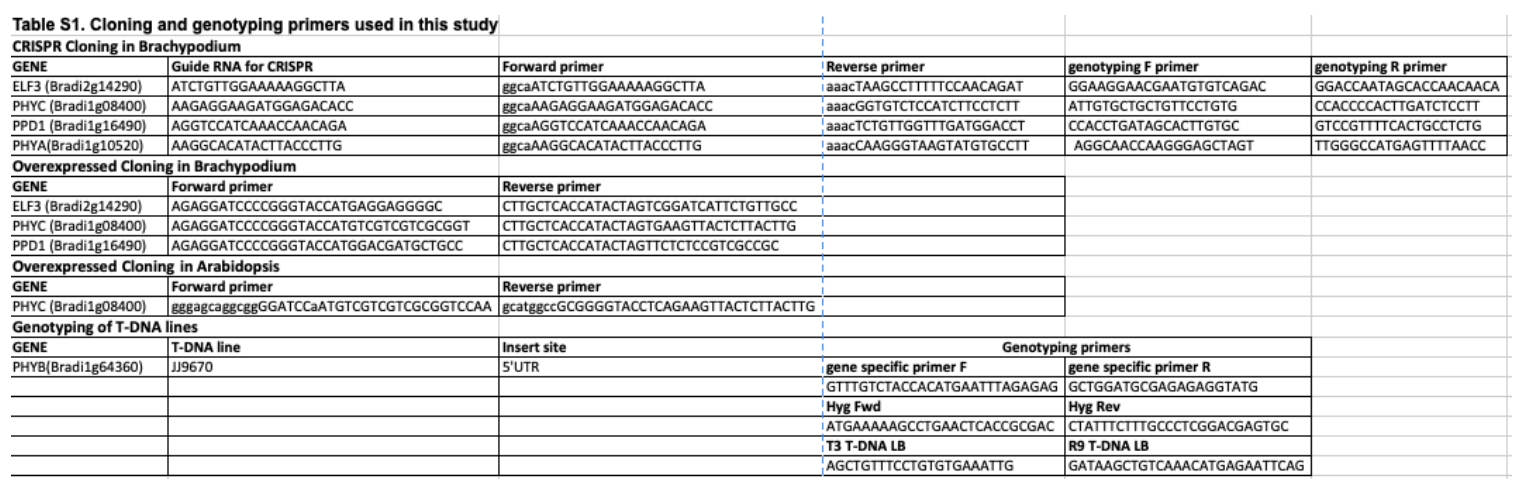

For Western blot assay, seeds were sterilized and sown on $1 / 2 \mathrm{X}$ Murashige and Skoogagar (MS-agar) plates at $\mathrm{pH} 5.7$ and grown in Magenta ${ }^{\mathrm{TM}}$ GA-7 Plant Culture Box (Thomas scientific). Sterilized seeds were stratified for 2 days at $4^{\circ} \mathrm{C}$ in the dark and allowed to germinate. The plates were transferred to short-day conditions (12 h light and $12 \mathrm{~h}$ dark) and collected at the indicated time.

3 plants per sample were grinded and $100 \mathrm{mg}$ of frozen plant material then added $100 \mu \mathrm{l}$ 2x Laemmli buffer (S3401, SIGMA). The protein was denatured at $96{ }^{\circ} \mathrm{C}$ for $10 \mathrm{~min} .15$ $\mu \mathrm{l}$ of protein samples were separated on $12 \%$ SDS-PAGE and blotted $7 \mathrm{~min}$ to nitrocellulose membrane using Turbo semi-dry transfer. Blots were blocked with $5 \%$ milk for $1 \mathrm{~h}$ at RT and then incubated in the anti-FLAG M2 (Sigma) primary antibody at a dilution of $1: 2500$ at $4{ }^{\circ} \mathrm{C}$ overnight with agitation. The antibody solution was decanted and the blot was rinsed briefly twice, then washed once for $15 \mathrm{~min}$ and 3 times for $5 \mathrm{~min}$ in TBS-T at RT with agitation. Blot was incubated in secondary antibody goat anti-mouse IgG-HRP conjugate (Bio-Rad, \#1721011) diluted to 1:5000 in for $2 \mathrm{~h}$ at RT with agitation. The blot was washed as above and developed by PiecreTM ECL substrate (Thermo Scientific, \#32134). Exposure time was 15 and 30 seconds.

\section{Gene expression by RNA-seq (Fig. 2)}

RNA-seq experiments were performed for Bd21-3, elf3-1, UBIpro:ELF3 and phyC-4, ppd-1, UBIpro:PPD1 at LD and SD over a 24h time course. 2, 3 or 6 week old seedlings of the indicated genotypes were grown at $20^{\circ} \mathrm{C}$ and sampled at intervals over the diurnal cycle: $\mathrm{ZT}=0,4,8,12,16,20$ and $22 \mathrm{~h}$. For each sample 3 leaves of 3 plants were mixed. The phyC-4 time course was done as replicate. In each time course performed a wildtype control included in all conditions and ZTs. 
Qiagen RNeasy Mini Kit (74104) was used to extract RNA. RNA quality and integrity were assessed on the Agilent 2200 TapeStation system. Library preparation was performed with $1 \mu \mathrm{g}$ total RNA using the NEBNext ${ }^{\circledR}$ Ultra $^{\mathrm{TM}}$ Directional RNA Library Prep Kit for Illumina ${ }^{\circledR}($ E7420L). The libraries were sequenced on a NextSeq500 (Illumina) running a final pooled library. Each pool contained 24 to 30 samples and was sequenced using NextSeq ${ }^{\circledR}$ 500/550 High Output Kit v2 (150 cycles) TG-160-2002 on a NextSeq500 (Illumina).

Q-PCR was performed on a Roche Lightcycler using standard reverse transcriptase kit and SYBR Green Real-Time PCR Master Mixes (SIGMA).

\section{RNA-Seq: Mapping and quantification (Fig. 2)}

Raw reads were mapped either with the Tophat pipeline or hisat $2+$ stringtie pipeline due to logistic reasons, and is recorded in the supplementary TPM table. We used "Bdistachyon_314_v3.1" from Phytozome (1) as reference genome throughout the study. Hisat2+Stringtie pipeline: Adapters were trimmed off from raw reads with Trimmomatic (2) with argument "ILLUMINACLIP:\$\{FA_ADAPTER $\}\}: 6: 30: 10$ LEADING:3 TRAILING:3 MINLEN:36 SLIDINGWINDOW:4:15". Raw reads were mapped to the transcriptome using HISAT2 (3) with argument:"--no-mixed --rnastrandness RF --dta --fr" . Duplicate reads were removed with Picard (4) using default setting. Transcripts were quantified according to this alignment with StringTie (5) in TPM values (Transcripts per Million mapped transcripts) with argument "--rf".

\section{Tophat pipeline}

The TPM values were transformed into log abundances through

$$
A_{g c}=\log _{2}\left(\mathrm{TPM}_{g c}+1\right)=A[c]
$$

( $g$ indexes genes while $c$ indexes conditions). 
Any gene with a maximum log abundance smaller than 3.0 was discarded from downstream analysis to avoid introducing noisy variation. Detailed TPM table can be found in the supplementary.

\section{RNA-Seq: Clustering and target calling}

To investigate transcriptomic response towards a particular treatment, time-course perturbation matrices were constructed as the difference of log abundances between paired conditions. For example,

$$
\begin{aligned}
D & {[\mathrm{LD} / \mathrm{SD}, \mathrm{WT}, \mathrm{Wk} 2, \mathrm{ZT} 0] } \\
& =A[\mathrm{LD}, \mathrm{WT}, \mathrm{Wk} 2, \mathrm{ZT} 0]-A[\mathrm{SD}, \mathrm{WT}, \mathrm{Wk} 2, \mathrm{ZT} 0] \\
& =\log _{2}\left(\frac{\mathrm{TPM}[\mathrm{LD}, \mathrm{WT}, \mathrm{Wk} 2, \mathrm{ZT} 0]+1}{\mathrm{TPM}[\mathrm{SD}, \mathrm{WT}, \mathrm{Wk} 2, \mathrm{ZT} 0]+1}\right)
\end{aligned}
$$

Clustering: The selected perturbation matrices were concatenated into a data matrix, against which a von Mises-Fisher mixture of increasing concentration is fitted (similar to clusterVMF (6)). Optimal concentration is manually selected to be $\sim 3.080$ according to the diagnostic plot and clusters with an average uncertainty higher than 2.5 were considered non-significant.

The following perturbation matrices were selected:

- $\quad[\mathrm{LD} / \mathrm{SD}, \mathrm{WT}, \mathrm{Wk} 2]$

- $\quad[\mathrm{LD} / \mathrm{SD}, \mathrm{WT}, \mathrm{Wk} 3]$

- $\quad[\mathrm{SD}$, elf3-1/WT,Wk2]

- $\quad[\mathrm{SD}$, elf3-1/WT,Wk3]

- $\quad[\mathrm{LD}, \mathrm{phyC}-4 / \mathrm{WT}, \mathrm{Wk} 2]$

- $\quad[\mathrm{LD}, \mathrm{ppd} 1-1 / \mathrm{WT}, \mathrm{Wk} 3]$

Selection of genes responsive to elf3-1 and phyC-4: A continous timecourse function was interpolated linearly from each of the perturbation matrices [SD, elf3-1/WT,Wk2] and [LD, phyC-4/WT,Wk2], which is then integrated over $24 \mathrm{~h}$ to give a temporal average, $T[\mathrm{SD}$, elf3-1/WT, Wk2] and $T[\mathrm{LD}$, phyC-4/WT, Wk2]. Genes that satisfy 
$T[\mathrm{SD}$, elf3-1/WT, Wk2 $]-T[\mathrm{LD}$, phyC-4/WT, Wk2 $]>1.0$ were considered transcriptionally perturbed.

\section{ChIP-seq experimental details (Fig. 3 and 4)}

Seeds were sterilized and sown on $1 / 2 \mathrm{X}$ Murashige and Skoog-agar (MS-agar) plates at pH 5.7 and grown in Magenta ${ }^{\mathrm{TM}}$ GA-7 Plant Culture Box (Thomas scientific) and harvested at the indicated time.

$3 \mathrm{~g}$ seedlings for each set were fixed under vacuum for $20 \mathrm{~min}$ in $1 \mathrm{xPBS}\left(10 \mathrm{mM} \mathrm{PO}_{4}{ }^{3-}\right.$, $137 \mathrm{mM} \mathrm{NaCl}$, and $2.7 \mathrm{mM} \mathrm{KCl}$ ) containing 1\% Formaldehyde (F8775 SIGMA). The reaction was quenched by adding glycine to a final concentration of $62 \mathrm{mM}$. Chromatin immunoprecipitation (ChIP) was performed as described (7), with the exception that 100 $\mu 1$ FLAG M2 agarose affinity gel antibody was used (SIGMA-Aldrich) per sample. Sequencing libraries were prepared using TruSeq ChIP Sample Preparation Kit (Illumina IP-202-1024) and samples sequenced on NextSeq500 machine from Illumina using NextSeq ${ }^{\circledR}$ 500/550 High Output Kit v2 (75 cycles) TG-160-2005. Sequence reads were analysed using in-house pipelines.

\section{ChIP-Seq: Mapping and quantification}

Adapters were trimmed off from raw reads with Trimmomatic (2) with argument "ILLUMINACLIP:\$\{\{FA_ADAPTER $\}\}: 6: 30: 10$ LEADING:3 TRAILING:3

MINLEN:36 SLIDINGWINDOW:4:15". Raw reads were mapped to the genome "Bdistachyon_314_v3.1" with Bowtie2 (7) under argument:"--no-mixed --no-discordant -no-unal -k2". Any read that mapped to more than one genomic location was discarded. Duplicate reads were removed with Picard using default setting (4).

Genomic binding profile was quantified in RPKM (Reads Per Kilobase per Million mapped reads) using a bin-size of $10 \mathrm{bp}$. For each bin,

$\mathrm{RPKM}_{\text {bin }}=\frac{\text { \#Reads covering bin }}{\text { bin-size }} \cdot \frac{10^{6}}{\text { \#Mapped reads }}$

For each treated ChIP-Seq library, peaks were called against a control using MACS2 ( 8 ) with argument "--keep-dup 1 -p 0.1". Peaks from all ChIP-Seqs were fitlered for FC $>2.0$. 


\section{ChIP-Seq: Defining regions differentially bound by ELF3}

Peaks from all ELF3 ChIP-Seqs were filtered and merged into contiguous regions.

Abundances were computed for 100bp windows within each merged region, and logtransformed with

$$
A_{w c}=A_{w}[c]=\log _{2}\left(1+\sum_{i \in w} \operatorname{RPKM}_{i}[c]\right)
$$

( $w$ denotes the window, $i$ denotes the $10 \mathrm{bp}$ bin, $c$ indexes condition)

Differential occupancy was quantified for each window by taking its dot-product with a signature vector to get $s_{w}=\sum_{c} \phi_{c} A_{w c}$, where the signature vector $\phi_{c}$ is computed from a set of marker windows $W$ so that

$$
\left\{\begin{array}{c}
\phi_{c}=\operatorname{meanNorm}_{c}\left(\frac{\sum_{w \in W} A_{w c}}{\sum_{w \in W} 1}\right) \\
\operatorname{meanNorm}_{c}\left(x_{c}\right)=x_{c}-\frac{\sum_{c} x_{c}}{\sum_{c} 1}
\end{array}\right.
$$

Cross-condition variance is also defined for each window $t_{w}=\operatorname{Std}_{c}\left[A_{w c}\right]$. Any window with $s_{g}>1.5$ and $t_{g}>0.5$ is called differentially bound.

Any peak with a differentially bound window is considered a differentially-bound peak. Any gene with a differential bound window within $+/-6000 \mathrm{bp}$ of its start codon is considered a differentially bound gene. Pile-up of ELF3 occupancy: was performed on the set of peaks that contains at least 1 differentially bound window.

ChIP-Seq: Peak-level overlap

Each ELF3 peak was considered overlapped if it's within 1000bp of a phyC peak, and vice versa. The venn is constructed by specifying the number of non-overlapping ELF3 
and phyC peaks, and the number of intersection peaks as (\#ELF3 peaks overlapped + \#phyC peaks overlapped) $/ 2$.

\section{Availability:}

Code is available from Github repo.

RNA-Seq data are available from: $\{\{\mathrm{GEO}$ RNA $\}\}$

ChIP-Seq data are available from: $\{\{$ GEO_CHIP $\}\}$

GNU-parallel (11) was used in paralleling the computational analysis.

Binding profiles from ChIP-Seq near marker genes were visualised with fluff ( 9 ), and visualised with Integrated Genomic Browser during investigation (10).

\section{Assaying dark reversion rate for PHYC (Fig. 4)}

\section{Lines used:}

B. distachyon

- WT

- $\quad$ phyC-ox in WT background; PUB2-PHYC-ox 19-7 (homozygous)

A. thaliana expressing $B d P H Y C$ in a phy $A B$ mutant background (plant 5)

\section{Method:}

B. distachyon seeds were incubated between 2 sheets of wet filter paper for 2-3 days in darkness at $4{ }^{\circ} \mathrm{C}$. After removal of the lemma, the seeds were plated on $1 / 2 \mathrm{MS}$ agar supplemented with $5 \mu \mathrm{M}$ Norflurazon to inhibit greening during the red light irradiation. The seedlings were grown for 6 days at $22{ }^{\circ} \mathrm{C}$ in darkness. In order to induce the degradation of BdphyA and BdphyB the seedlings were irradiated with constant red light (660 nm, $10 \mu \mathrm{mol} \mathrm{m} \mathrm{m}^{-2} \mathrm{~s}^{-1}$ ) for $16 \mathrm{~h}$. Subsequently, the seedlings were transferred to darkness at $22{ }^{\circ} \mathrm{C}$ to monitor dark reversion of BdphyC. At time points $0,4,8$ and $12 \mathrm{~h}$ after dark transfer relative levels of active BdphyC (Pfr/Ptot) were measured using a dual wavelength ratio spectrophotometer (Ratiospect) as described previously (8). The shoot parts of 5-7 B. distachyon seedlings were used per measurement. To inhibit oxidation, the seedlings were incubated for $20 \mathrm{~min}$ in ice-cold $50 \mathrm{mM}$ DTT solution prior to the measurement. 
A. thaliana seeds were sterilised before plating them on 4 layers of Whatman ${ }^{\circledR}$ filter

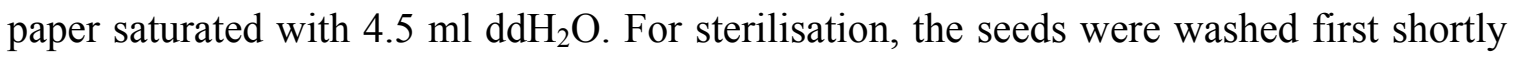
with $70 \%$ ethanol and then twice with $100 \%$ ethanol. The seeds were stratified for at least 2 days at $4{ }^{\circ} \mathrm{C}$ in darkness. To induce germination, the seeds were incubated during 4 to $8 \mathrm{~h}$ in white light at $22{ }^{\circ} \mathrm{C}$. Subsequently, the seedlings were grown in darkness at 22 ${ }^{\circ} \mathrm{C}$ for 4 days. Prior to Ratiospect measurements, the seedlings were irradiated for $20 \mathrm{~min}$ with constant red light $\left(660 \mathrm{~nm}, 10 \mu \mathrm{mol} \mathrm{m}^{-2} \mathrm{~s}^{-1}\right)$ to convert BdphyC into the active Pfr form. Afterwards the seedlings were transferred into darkness darkness at $22{ }^{\circ} \mathrm{C}$ to monitor dark reversion of BdphyC. At time points $0,4,8$ and $12 \mathrm{~h}$ after red light irradiation relative levels of active BdphyC (Pfr/Ptot) were measured using a dual wavelength ratio spectrophotometer (Ratiospect) as described previously (8). 120-140 mg of $A$. thaliana seedlings (freshweight) were used per measurement.

\section{Proteomics}

Plant materials for affinity purification coupled with mass spectrometry (AP-MS) were made from Brachypodium plants expressing either pUBI-ELF3-GFP-FLAG or pMDC32YFP-His $_{6}-3 x$ FLAG (negative control) transgene. After stratification in dark at $4{ }^{\circ} \mathrm{C}$ for 4 days, transgenic Brachypodium plants were grown in a growth chamber with a photoperiod of 14 hours of light $\left(200 \mathrm{umol} \cdot \mathrm{m}^{-2} \cdot \mathrm{s}^{-1}\right)$ and 10 hours of darkness, at $24{ }^{\circ} \mathrm{C}$ during daytime and $18{ }^{\circ} \mathrm{C}$ at night. Leaves from 45-day-old (old) or 21-day-old (young) plants were harvested at ZT0 in darkness and flash frozen in liquid $\mathrm{N}_{2}$. The protein extraction was performed in darkness with dim green safety light. About $30 \mathrm{mg}$ (for old plants sample and YFP negative control) or $10 \mathrm{mg}$ (for young plants sample) of total protein were used for purification via FLAG immune-precipitation (we used $1.4 \mu \mathrm{g}$ antiFLAG antibody per $1 \mathrm{mg}$ total protein), using the method as previously described $(6,7)$. After elution with $3 \times$ FLAG free peptides, eluates were precipitated by $25 \%$ TCA at -20 ${ }^{\circ} \mathrm{C}$, pelleted and washed with ice-cold acetone. Pellets were dried using a speed vacuum and sent for mass spectrometry analysis, with the same processing protocol and filtering criteria as described previously (8). MS data were extracted and searched against Brachypodium database to identify each protein (Phytozome 12, https://phytozome.jgi.doe.gov/pz/portal.html). All proteins identified in YFP control were 
subtracted from the identifications and a curated list containing BdELF3 specific interactors was presented, showing names of their Arabidopsis homolog proteins. log file of ChIP-Seq 188CR:

S1:

64-LD-ZT0-ELF3-OX-RERUN_S1.bowtie2

56859885 reads; of these:

56859885 (100.00\%) were paired; of these:

10994569 (19.34\%) aligned concordantly 0 times

24159948 (42.49\%) aligned concordantly exactly 1 time

$21705368(38.17 \%)$ aligned concordantly $>1$ times

$80.66 \%$ overall alignment rate

S2:

64-SD-ZT0-ELF3-OX-RERUN_S2.bowtie2

32907407 reads; of these:

32907407 (100.00\%) were paired; of these:

12224010 (37.15\%) aligned concordantly 0 times

11387902 (34.61\%) aligned concordantly exactly 1 time

9295495 (28.25\%) aligned concordantly $>1$ times

$62.85 \%$ overall alignment rate

S3:

64-LL-ZT0-ELF3-OX-RERUN_S3.bowtie2

47414081 reads; of these:

47414081 (100.00\%) were paired; of these:

12398525 (26.15\%) aligned concordantly 0 times

18170203 (38.32\%) aligned concordantly exactly 1 time

$16845353(35.53 \%)$ aligned concordantly $>1$ times

$73.85 \%$ overall alignment rate

S4:

64-LD-ZT4-ELF3-OX-RERUN_S4.bowtie2

44936828 reads; of these:

44936828 (100.00\%) were paired; of these: 
12788499 (28.46\%) aligned concordantly 0 times

18976870 (42.23\%) aligned concordantly exactly 1 time

13171459 (29.31\%) aligned concordantly $>1$ times

$71.54 \%$ overall alignment rate

S5:

64-SD-ZT4-ELF3-OX-RERUN_S5.bowtie2

44752906 reads; of these:

44752906 (100.00\%) were paired; of these:

16478316 (36.82\%) aligned concordantly 0 times

15698163 (35.08\%) aligned concordantly exactly 1 time

$12576427(28.10 \%)$ aligned concordantly $>1$ times

$63.18 \%$ overall alignment rate

S6:

66-LD-ZT4-PRR37OX-RERUN_S6.bowtie2

44752906 reads; of these:

44752906 (100.00\%) were paired; of these:

16478316 (36.82\%) aligned concordantly 0 times

15698163 (35.08\%) aligned concordantly exactly 1 time

$12576427(28.10 \%)$ aligned concordantly $>1$ times

$63.18 \%$ overall alignment rate

S7

64-LD-ZT20-ELF3OX-RERUN_S7.bowtie2

54058806 reads; of these:

54058806 (100.00\%) were paired; of these:

15052008 (27.84\%) aligned concordantly 0 times

$21878336(40.47 \%)$ aligned concordantly exactly 1 time

17128462 (31.68\%) aligned concordantly $>1$ times

$72.16 \%$ overall alignment rate

S8:

64-SD-ZT20-ELF3OX-RERUN_S8.bowtie2

63445898 reads; of these: 
63445898 (100.00\%) were paired; of these:

14483986 (22.83\%) aligned concordantly 0 times

$28850417(45.47 \%)$ aligned concordantly exactly 1 time

20111495 (31.70\%) aligned concordantly $>1$ times

$77.17 \%$ overall alignment rate

S9:

phyC-OX-GFP-FLAG-Zt20-LD-RERUN_S9.bowtie2

49021059 reads; of these:

49021059 (100.00\%) were paired; of these:

11516381 (23.49\%) aligned concordantly 0 times

17325692 (35.34\%) aligned concordantly exactly 1 time

$20178986(41.16 \%)$ aligned concordantly $>1$ times

$76.51 \%$ overall alignment rate

S10:

phyC-OX-GFP-FLAG-Zt20-LD-RERUN_S10.bowtie2

43207042 reads; of these:

43207042 (100.00\%) were paired; of these:

$9431466(21.83 \%)$ aligned concordantly 0 times

17663422 (40.88\%) aligned concordantly exactly 1 time

16112154 (37.29\%) aligned concordantly $>1$ times

$78.17 \%$ overall alignment rate

175C run_INPUT-BD_S4

22572371 reads; of these:

22572371 (100.00\%) were unpaired; of these:

$1604341(7.11 \%)$ aligned 0 times

12073412 (53.49\%) aligned exactly 1 time

8894618 (39.40\%) aligned $>1$ times

$92.89 \%$ overall alignment rate 


\section{REFERENCES}

1. D. P. Woods, T. S. Ream, G. Minevich, O. Hobert, R. M. Amasino, Genetics, in press, doi:10.1534/genetics.114.166785.

2. J. Miao et al., Targeted mutagenesis in rice using CRISPR-Cas system. Cell Res. 23, 1233-6 (2013).

3. S. C. Alves et al., A protocol for Agrobacterium-mediated transformation of Brachypodium distachyon community standard line Bd21. Nat Protoc. 4, 638-649 (2009).

4. H. Huang et al., PCH1 integrates circadian and light-signaling pathways to control photoperiod-responsive growth in Arabidopsis. Elife. 5 (2016), doi:10.7554/eLife.13292.

5. M. D. Curtis, U. Grossniklaus, A Gateway Cloning Vector Set for HighThroughput Functional Analysis of Genes in Planta. PLANT Physiol. 133, 462469 (2003).

6. J. Vogel, T. Hill, High-efficiency Agrobacterium-mediated transformation of Brachypodium distachyon inbred line Bd21-3. Plant Cell Rep. 27, 471-478 (2008).

7. K. E. Jaeger, N. Pullen, S. Lamzin, R. J. Morris, P. A. Wigge, Interlocking feedback loops govern the dynamic behavior of the floral transition in Arabidopsis. Plant Cell. 25, 820-33 (2013).

8. J. Rausenberger et al., An integrative model for phytochrome B mediated photomorphogenesis: From protein dynamics to physiology. PLoS One. 5 (2010), doi:10.1371/journal.pone.0010721.

9. H. Huang, D. Nusinow, Tandem Purification of His6-3x FLAG Tagged Proteins for Mass Spectrometry from Arabidopsis. BIO-PROTOCOL. 6 (2016), doi:10.21769/BioProtoc.2060.

10. H. Huang et al., Identification of Evening Complex Associated Proteins in Arabidopsis by Affinity Purification and Mass Spectrometry. Mol. Cell. Proteomics. 15, 201-217 (2016).

11. J. A. Vizcaíno et al., ProteomeXchange provides globally coordinated proteomics data submission and dissemination. Nat. Biotechnol. 32, 223-6 (2014). 